Noname manuscript No.

(will be inserted by the editor)

\section{Progress and Prospects of the Human-Robot Collaboration}

\author{
Arash Ajoudani, Andrea \\ Maria Zanchettin, Serena \\ Ivaldi, Alin Albu-Schäffer, \\ Kazuhiro Kosuge, and \\ Oussama Khatib
}

Received: date / Accepted: date

\begin{abstract}
Recent technological advances in hardware design of the robotic platforms enabled the implementation of various control modalities for improved interactions with humans and unstructured environments. An important application area for the integration of robots with such advanced interaction capabilities is human-robot collaboration. This aspect represents high socio-economic impacts and maintains the sense of purpose of the involved people, as the robots do not completely replace the humans from the work process. The research community's recent surge of interest in this area has been devoted to the implementation of various methodologies to achieve intuitive and seamless humanrobot-environment interactions by incorporating the collaborative partners' superior capabilities, e.g. human's cognitive and robot's physical power generation capacity. In fact, the main purpose of this paper is to review the state-of-theart on intermediate human-robot interfaces (bi-directional), robot control modalities, system stability, benchmarking and relevant use cases, and to extend views on the required future developments in the realm of human-robot collaboration.
\end{abstract}

Arash Ajoudani is with $\mathrm{HRI}^{2}$ Lab of the Istituto Italiano di Tecnologia, Genoa, Italy, Email: arash.ajoudani@iit.it

Andrea Maria Zanchettin is with Politecnico di Milano, Dipartimento di Elettronica, Informazione e Bioingegneria, Milano, Italy, Email: andreamaria.zanchettin@polimi.it

Serena Ivaldi is with INRIA Nancy Grand-Est, France, and the Intelligent Autonomous Systems Lab of TU Darmstadt, Germany. Email: serena.ivaldi@inria.fr

Alin Albu-Schäffer is with the Institute of Robotics and Mechatronics, German Aerospace Center (DLR), Germany, Email: Alin.AlbuSchaeffer@dlr.de

Kazuhiro Kosuge is with the System Robotics Laboratory, Tohoku University, Japan, Email: kosuge @m.tohoku.ac.jp

Oussama Khatib is with the Stanford Robotics Laboratory, Stanford University, USA, Email: ok@ robo.stanford.edu

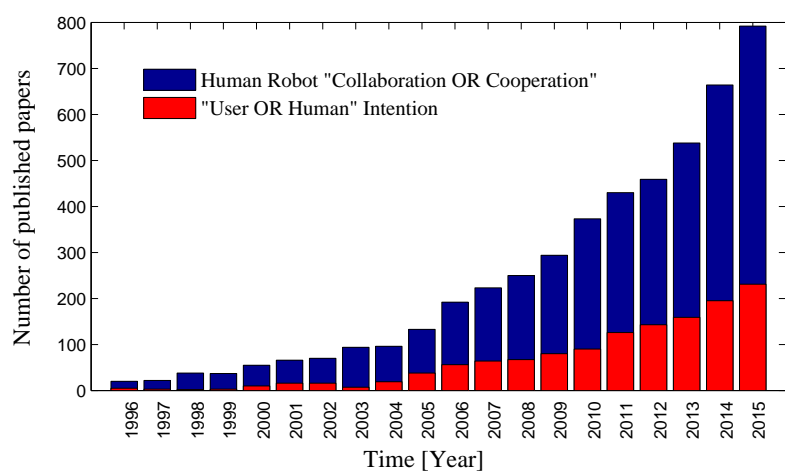

Fig. 1: Number of publications on the topic of human-robot collaboration from 1996 to 2015. The contribution of the research keyword "human intention" (red) to the numbers is illustrated in this plot. The data is extracted from Google Scholar.

\section{Introduction}

The fast growing demands for service robot applications in home or industrial workspaces have led to the development of several well-performing robots equipped with rich proprioception sensing and actuation control. Such systems that range from robotic manipulators [Albu-Schäffer et al., 2007] to full humanoids [Tsagarakis et al., 2016, Ott et al., 2006, Kaneko et al., 2008, Radford et al., 2015] are expected to help the human user in various tasks, some of which require collaborative effort for a safe ${ }^{1}$, successful, and time and energy efficient execution. In fact, the integration of robotic systems in collaborative scenarios has seen an extensive and fast-growing research effort, a tentative estimation of which is provided in Fig. 1 by referring to the number of publications on this topic over the last two decades.

Physical human-robot collaboration (PHRC), which falls within the general scope of physical human-robot interaction (see [De Santis et al., 2008, Murphy, 2004, Alami et al., 2006]), is defined when human(s), $\operatorname{robot}(s)$ and the environment come to contact with each other and form a tightly coupled dynamical system to accomplish a task [Bauer et al., 2008, Krüger et al., 2009]. Ideally, each active component of such a system must be capable of observing and estimating the counterparts' contributions to the overall system's response through the fusion and processing of the sensory information [Argall and Billard, 2010, Ebert and Henrich, 2002, Lallée et al., 2012]. As a consequence, an appropriate reactive behaviour can be replicated (e.g. by the human from a set of obtained skills in previous attempts of performing a similar task) or developed to complement and improve the performance of the collaborative partners.

\footnotetext{
1 The problem of safety in human-robot interaction (HRI) and the related open issues have been extensively discussed in literature [Haddadin et al., 2009, De Santis et al., 2008, Alami et al., 2006]. Hence, our focus in this review paper will be on other important aspects of physical human-robot collaboration (PHRC).
} 
Similar to the humans' anticipatory (feed-forward [Shadmehr and Mussa-Ivaldi, 1994b]) or feed-back [Todorov and Jordan, 2002] mechanisms to develop a suitable motor behaviour, the collaborative robots' response to sensory input can be achieved through model/knowledge based techniques [Tamei and Shibata, 2011, Ogata et al., 2003, Kimura et al., 1999, Magnanimo et al., 2014], the implementation of feedback controllers with pre-set interaction modalities [Peternel et al., 2016c, Donner and Buss, 2016a] or a combined approach [Rozo et al., 2013, Peternel et al., 2016b, Lawitzky et al., 2012b, Palunko et al., 2014]. A key strategy in this direction is the establishment of a shared authority framework in which the significant capabilities of both humans and robots can be exploited. For instance, humans' significant cognitive abilities in learning and adaptation to various tasks demands and disturbances can be used to supervise the collaborative robots' superior physical capabilities. The increasing slope in the research community's interest towards the integration of human intention in real-time adaptation of the robot behaviour is illustrated in Fig.1.

With this in mind, the main purpose of this paper is to review the state-of-the-art on the key enabling technologies to achieve a seamless and intuitive human-robot collaboration. Although hardware-related components are among the most critical to achieve this goal, a remarkable effort has been recently devoted to overview the underlying progress in terms of communication [Wang et al., 2005], sensing [Tegin and Wikander, 2005], and actuation [Vanderborght et al., 2013a] developments. Hence, our focus in this review will be on other relevant key elements of the HRC, i.e. human-robot interfaces (bi-directional), robot control modalities, system stability, benchmarking and relevant use cases. In addition, with the aim to achieve a reasonable convergence of views for the required future developments, our focus will be mostly on the physical aspects ${ }^{2}$ of the human-robot collaboration.

\section{Interfaces for Improved Robot Perception}

Humans embrace a diversity of experiences from working together in pairs or groups. This has contributed to the development of implicit and explicit communication standards so that task-related information can be perceived and communicated intuitively [Sebanz et al., 2006]. In fact, one of the main objectives in the realm of physical human-robot collaboration is to design and establish similar communication standards so that the robot is aware of human intentions and needs in various phases of the collaborative task [Klingspor et al., 1997, Bauer et al., 2008]. Despite the fact that the robotic replication of the human sensory system is hardly

\footnotetext{
2 A good overview of the cognitive aspects in HRC can be found in the literature, e.g., see [Fong et al., 2003, Freedy et al., 2007, Rani et al., 2004]
}

possible with the current technology, the understanding and implementation of the underlying communication principles can potentially lead to an enhanced physical human-robot interaction performance [Reed and Peshkin, 2008].

A widely known example of such a communication interface is built on the use of visual [Perzanowski et al., 1998, Agravante et al., 2014a, Morel et al., 1998] or language commands [Medina et al., 2012a, Miyake and Shimizu, 1994,Petit et al., 2013], as user-friendly means of communicating with a robot, from the human standpoint, given that the human is not required to learn additional tools. The use of head, body or arm gestures are common examples in the areas of human-robot interaction and collaboration [Li et al., 2005, Carlson and Demiris, 2012]. In this direction a method to interpret the human intention from the latest history of the gaze movements and to generate an appropriate reactive response in a collaborative setup was proposed in [Sakita et al., 2004]. Authors in [Hawkins et al., 2013] developed a vision-based interface to predict in a probabilistic manner when the human will perform different subtasks that may require robot assistance. The developed technique allows for the tracking of the human variability, environmental constraints, and task structure to accurately analyse the timings of the human partner's actions.

Although such interfaces appear natural to the humans, their usage is mostly limited to activating high-level robot operations and the task complexity can potentially prevent the robot from deriving the desired sensorimotor behaviour from these higher-level modalities. In fact, a large degree of robot autonomy, which is far beyond current capabilities of the autonomous robots, is required for vision or auditory based interfaces to function on a wide range of applications.

An alternative approach to the design of human-robot interfaces recognises the use of force/pressure sensors in contact to anticipate the objective of the human partner and/or to control the cooperation effort. Due to the simplicity of the underlying mechanism, it has been explored in several applications, examples include collaborative object transportation [Ikeura and Inooka, 1995a,Kosuge and Kazamura, 1997a, Al-Jarrah and Zheng, 1997a,Tsumugiwa et al., 2002a,Duchaine and Gosselin, 2007, Agravante et al., 2014a, Gribovskaya et al., 2011a, Rozo et al., 2014, Rozo et al., 2015, Adams et al., 1996], object lifting [Evrard and Kheddar, 2009,Evrard et al., 2009], object placing [Tsumugiwa et al., 2002a, Gams et al., 2014], object swinging [Donner and Buss, 2016a,Palunko et al., 2014], posture assistance [Ikemoto et al., 2012, Peternel and Babič, 2013], and industrial complex assembly processes [Krüger et al., 2009] (see also Fig. 2).

In most of the above techniques, the interaction forces/torques are used to regulate the robot control parameters and trajectories following the admittance [Duchaine and Gosselin, 2009, Lecours et al., 2012] or impedance [Tsumugiwa et al., 2002a,Agravante et al., 2014a] causality [Hogan, 1985]. Notwith- 


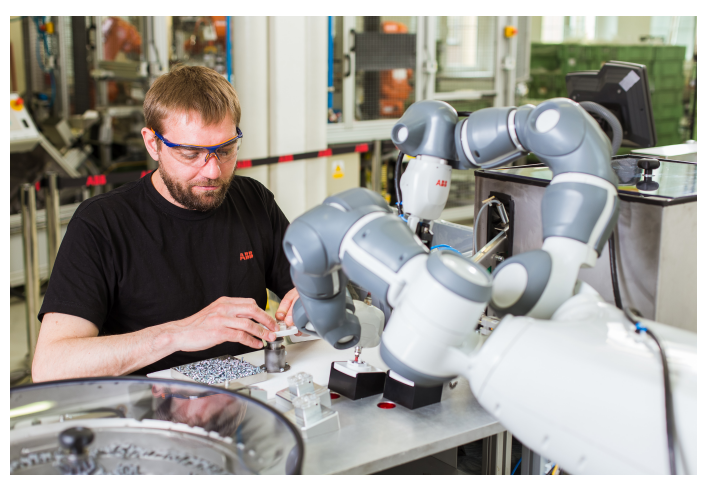

Fig. 2: An example of human-robot collaborative manipulation in a productive environment (image courtesy of $\mathrm{ABB}$ received from www.abb.cz).

standing the wide margin of applications, collaborative tasks that involve simultaneous interaction with rough or uncertain environments (e.g. co-manipulative tool-use) can induce various unpredictable force components to the sensor readings [Peternel et al., 2014]. This can significantly reduce the suitability of such an interface in more complex interaction scenarios since it can be difficult to distinguish the components related to the active counterpart(s) behaviour from the ones generated from the interaction with the environment.

Bio-signals such as electromyography (EMG) and electroencephalography (EEG), or other physiological indices such as electrodermal activity [Pecchinenda, 1996, Rani et al., 2006] can be used to anticipate the human intention in PHRC. In particular, due to the adaptability and ease-of-use of EMG measurements, they have found a wide range of applications in human-in-the-loop robot control such as: prosthesis [Farry et al., 1996, Jiang et al., 2009, Farina et al., 2014, Castellini et al., 2014, Strazzulla et al., 2017], exoskeletons [Rosen et al., 2001, Fleischer and Hommel, 2008] and industrial manipulator control [Vogel et al., 2011, Peternel et al., 2014, Ajoudani, 2016, Gijsberts et al., 2014]. Peternel et al., used EMG signals to anticipate the stiffening/complying behaviour of a torque controlled robotic arm in a co-manipulation task [Peternel et al., 2016c]. Through this interface, the leading/following roles of human and the robot counterpart were estimated online. In another study, Bell et al., used EEG signals to command a partially autonomous humanoid robot through high-level descriptions of the task [Bell et al., 2008].

A remarkable use of bio-signals in the development of HR interfaces is to estimate the human physical (e.g. fatigue) or cognitive (anxiety, in-attention, etc.) state variations that might deteriorate the collaborative robot'(s) performance. The authors in [Rani et al., 2004] developed a method to detect human anxiety in a collaborative setup by extracting features from EMG, electrocardiography (ECG) and Electrodermal responses. In a similar work, the human physical fatigue was detected and used to increase the robot's contribution to the task execution [Peternel et al., 2016b].

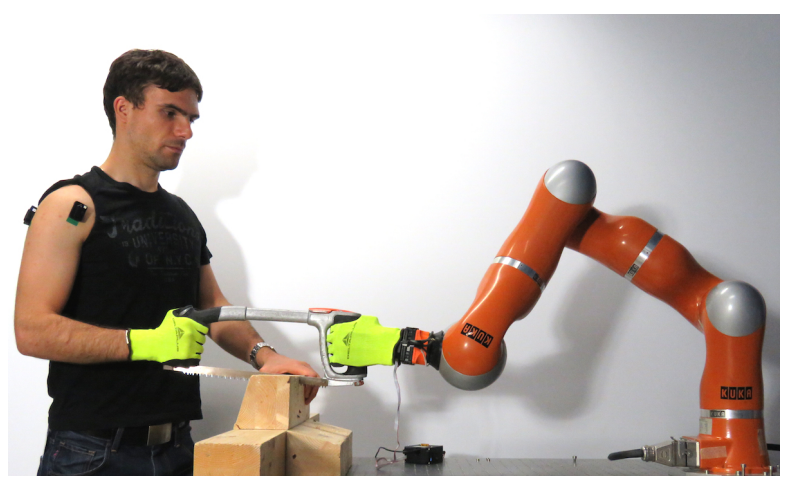

Fig. 3: Authors in [Peternel et al., 2016b] proposed a human-robot comanipulation framework for robot adaptation to human fatigue. The myoelectric interface provides the robot controller with a feedback about human motor behaviour to achieve an appropriate impedance profile in different phases of the task. The human fatigue estimation system provides the robot with the state of the human physical endurance (image courtesy of L. Peternel).

Although an interface that is build on a unique source of sensory data can configure a pre-defined robot behaviour in collaborative settings, the underlying functionality is limited and cannot be easily generalised to cross domain scenarios. For instance, the use of visual feedback for the estimation of the exchanged amount of energy between the counterparts is less effective than the use of force or pressure sensors. Similarly, the use of bio-signals such as EMGs for tracking of the human limb movements may result in less accurate performances in comparison to the external optical or IMU-based (inertial measurement unit) tracking systems [Corrales et al., 2008]. To address this, a combined approach, associating multi-modal sensory information to different robot control modalities (commonly known as multimodal interfaces [Mittendorfer et al., 2015, Peternel et al., 2016c]), can be exploited. In this direction, the authors in [Agravante et al., 2014a] proposed a hybrid approach by merging vision and force sensing, to decouple high- and low-level interaction components in a joint transportation task where a human and humanoid robot carry a table with a freely moving ball on top (see also [Rozo et al., 2016]). A similar work proposed a multi-modal scheme for intelligent and natural human-robot interaction [Böhme et al., 2003] by merging vision-based techniques for user localisation, person localisation and person tracking and their embodiment into a multi-modal overall interaction schema.

In a similar fashion, voice commands were used to pause, stop or resume the execution of a dynamic co-manipulation task, the control parameters of which regulated by an EMG based interface (See Fig. 3). In this work, an external tracking system detected the human arm configuration to regulate the robot task frame in real-time. By the same token, authors in [Yang et al., 2016] developed a multi-modal teaching interface on a dual-arm robotic platform. The interface was built on the use of EMG on the user arm and force sensors on robot end-effector. In this setup, one robotic arm is 


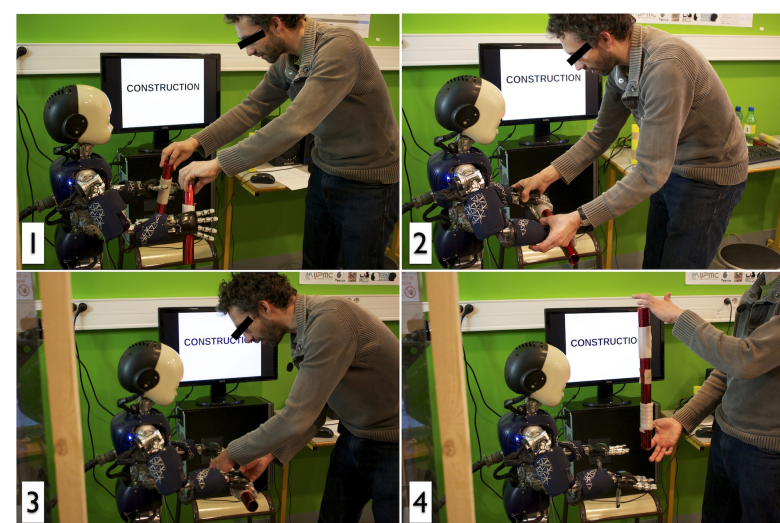

Fig. 4: In [Ivaldi et al., 2016] naive participants (not expert in robotics) interacted with the humanoid iCub to build an object: the physical interaction was at the level of the arms, covered by a soft tactile skin (image courtesy of S. Ivaldi).

connected to the tutee's arm providing guidance through a variable stiffness control approach, and the other to the tutor to capture the motion and to feedback the tutees performance in a haptic manner. The reference stiffness for the tutors arm stiffness was estimated in real-time and replicated by the tutee's robotic arm. Ivaldi et al., [Ivaldi et al., 2016] studied multi-modal communication of people interacting physically with the humanoid iCub to build objects (see Fig. 4). Participants would naturally gaze at the robot's hands or face to communicate the focus of attention of the collaborative action, while speaking to the robot to describe each action. The authors found that individual factors of the participants influence the production of referential cues, both in speech and gaze: particularly, people with negative attitude towards robots avoid gazing at the robot, while extroverted people speak more to the robot during the collaboration. The robot, controlled in impedance with a low stiffness at joints, switched to zero-torque control when the humans were grasping the robot arms covered by a tactile skin (enabling precise contact estimation [Fumagalli et al., 2012]) and giving the voice command to "be compliant". This multi-modal command strategy allowed the participants, not experts in robotics and mostly interacting with a robot for the first time, to physically move the robot arms in an intuitive way, without generating anxiety for their safety as reported by the participants in their interviews. Despite the lack of experience, all the human participants were able to interact physically with the robot to teach the task. Facilitated probably by the child-like appearance of the $\mathrm{iCub}$, the participants naturally acted as teachers/caregivers, in line with the observations of [Nagai and Rohlfing, 2009]; however, it has to be remarked that in that situation of physical interaction the authors did not observe exaggerated movements typical of parental motionese/scaffolding situations in HRI, where often there is little physical interaction and more social cues as gaze or gestures. These results are in accord with previous work presented, see e.g. [Kilner et al., 2003, Ugur et al., 2015].

The improved performance of the multi-modal interfaces in the generation of compound robot behaviours that are required to execute more complex collaborative tasks has shifted the attention towards the usage and fusion of multisource sensory information. Results of Google Scholar suggest that over $76 \%$ of the publications in the area of humanrobot collaboration used multi-modal interfaces in year 2015 . Nevertheless, the inclusion of more communication channels in the development of the intermediate interfaces will potentially contribute to an increase in the human cognitive burden and the low level robot control complexity. This may affect the intuitiveness of the interface and result in an excessive human effort to operate a specific robot modality. A solution to this issue can be obtained by the introduction of shared communication modalities [Green et al., 2008, Lackey et al., 2011,Lackey et al., 2011]. Alternatively, robotic learning techniques such as: gradual mutual adaptation [Ikemoto et al., 2012, Peternel et al., 2016a], reinforcement learning [Palunko et al., 2014] or learning from demonstration [Evrard et al., 2009, Lawitzky et al., 2012a, Rozo et al., 2015] can be exploited to weaken the communication loops' demands (e.g. bandwidth, number of feedback modalities) due to an increased level of robot autonomy.

\section{Interfaces for Improved Human Perception}

The visual and auditory systems of the humans provide powerful sensory inputs that contribute to a fast and accurate perception of the movement kinematics and the environment, and a constant update of the internal models. The role of such sensory inputs in dynamic perception of the environment, e.g. anticipating the weight of an object through vision [Gordon et al., 1993], and estimating a required amount of force to move the object along a pre-defined path [Johansson, 1998], has also been investigated.

During collaboration and dyadic interaction, mutual gaze and joint attention are common ways of conveying information [Tomasello, 2009]. Such mechanisms are often implemented in robots to make the interaction more effective by providing additional back-channels. For example in [Ivaldi et al., 2014] the robot was equipped with anticipatory gaze mechanisms and proactive behaviours, increasing the pace of the interaction and reducing the reaction time of the human to the robot's cues.

In a similar study, Dumora et al. [Dumora et al., 2012], studying haptic communication between an operator and a robot for a bar transportation task, observed that wrench measurements provide incomplete information to detect the operator's intent of motion. This has been also observed in [Reed, 2012] for cooperating dyads able to communicate 


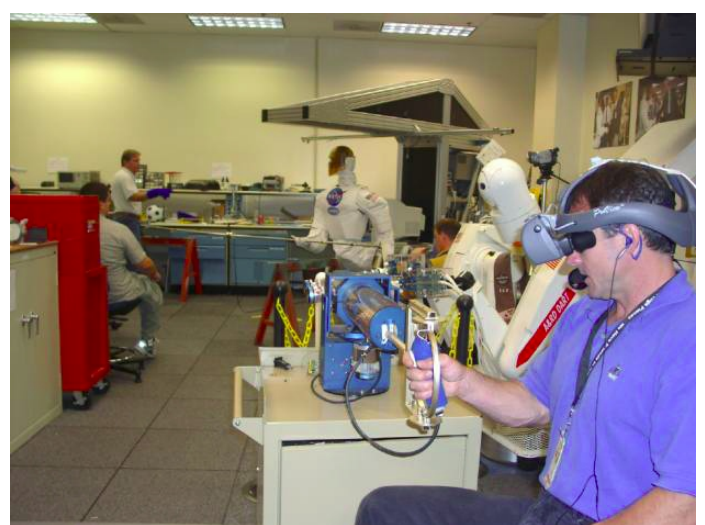

Fig. 5: An example of providing haptic and visual feedback (via AR) to the human counterpart in collaborative settings [Glassmire et al., 2004] (image courtesy of M. O’Malley).

only through physical interaction ${ }^{3}$. Although haptic feedback can temporarily be sufficient to achieve sub-tasks, if contextual information about the task phase are provided, for the interaction to be efficient more non-verbal cues are necessary to recognize the partner intent during collaborative tasks and synchronize the dyadic activity. In this sense, joint attention or proactivity can improve the mutual awareness, hence the task performance, as shown by [Ivaldi et al., 2014] for a dyadic learning task.

An enhanced perception of the environment through human visual feedback can also be achieved by Augmented Reality (AR) approach, enabling the human partner to observe and review a plan with the robot prior to execution [Green et al., 2009, Glassmire et al., 2004] (see Fig. 5). Head Mounted Displays, that were mostly used in research labs or in very specific, critical applications (e.g. aero-space and military), are nowadays becoming a consumer product: the Rift by Oculus and the Gear VR by Samsung (among others), are the first modern and large scale examples of a market that is on the verge of the explosion. Potential drawbacks of the AR based approaches can be the information overloading, limited privacy (e.g. augmenting without permission) and additional cost, that may limit the expected performance of such systems in collaborative settings.

Haptic information provided by receptors in human limbs (fingertips, arm skin, etc.), on the other hand, represents a very important and complementary input to explore the external environment and for everyday task accomplishments. Since in most collaborative scenarios the human partner comes to physical contact(s) with the object and/or the robot in a closed dynamic chain, a large amount of meaningful information can be perceived by the human receptors. Nevertheless, as regards specific interaction scenarios in which the human perception of the environment is influenced by the task or environmental conditions, the use of artificial sen-

\footnotetext{
3 The effect of human sensorimotor learning is very crucial in such scenarios as the perception of the environment and the task by two experts will be different from two naive operators.
}

sory systems in the provision of haptic feedback to the human partner can be beneficial.

Although replicating the richness of human sensory apparatus through artificial systems remains a daunting task, the state-of-art reviews several non-invasive techniques to present haptic stimuli to robot operators, by delivering different types of stimuli to the human limb. This is not only to provide cheaper and easily applicable feedback systems can replace full force feedback with none or small performance reduction, but also to resolve fundamental issues such as stability in closed loop [Tegin and Wikander, 2005]. In addition, such sensory substitution techniques can also be used to provide the human with feedback about the advanced robotic perception of the environment (e.g., depth sensors [Plagemann et al., 2010]), that may not be covered by the human sensory system. Examples include vibrotactile, electrocutaneous or mechanical pressure [Shannon, 1976, Nohama et al., 1995, Wang et al., 1995], to convey information about force, proprioception, and/or texture. By the same token, previous works recognized different types of non-invasive sensory substitution techniques, roughly dividing haptic information into low-frequency, e.g. force related and highfrequency [Ajoudani et al., 2014], e.g. acceleration related [Godfrey et al., 2013]. In a similar vein, a haptic force feedback interface was developed in [Yang et al., 2016] to provide a tutor with information about the deviations of the tutee arm from desired trajectories in a human-robot collaboration setup that was developed as a teaching interface through a dual-arm robotic platform. The amount of force was related to the kinematic error between the desired and the tutee's replicated trajectories in real-time.

Table 1 presents an overview of the interfaces used in literature with the purpose of enhancing the robot and human perception of the task and the environment.

\section{Interaction Modalities}

This section aims at presenting an overview of the different strategies to endow the robot with interaction capabilities. While the interfaces and the underlying perception mechanisms are dealt with in Section 2, this part discusses different approaches to link the perception inputs to their actual effects in terms of robot behaviour.

As mentioned earlier, a very important aspect in setting up a successful PHRC framework is to achieve robot adaptivity to the task conditions and environmental constraints. One of the pioneering works in this sense are due to Hogan [Hogan, 1985], presenting the well-known impedance (and admittance, as a dual representation) control framework. Although the original work did not cover explicitly the case of human-robot interaction, the same formalism has been further developed in [Kosuge and Kazamura, 1997b, Tsumugiwa et al., 2002b, Albu-Schäffer et al., 2007, Albu-Schäffer et al., 
2007, Gribovskaya et al., 2011b] to explicitly account for the human as the source of interaction forces. In particular, Kosuge et al. proposed several control strategies to allow the human and a robotic manipulator to co-manipulate heavy and bulky objects in [Kosuge and Kazamura, 1997b]. In this scenario, that is quite common in the literature, the robot is responsible for carrying a payload, while the human steers the object to a desired position or along a desired path.

A similar concept was investigated in [Tsumugiwa et al., 2002b], where the authors augmented the sensor space with virtual forces to ease the human to complete a collaborative peg-in-hole task. The work in [Bestick et al., 2015] presented a framework for the parameter and state estimation of personalised human kinematic models using motion capture data. Data-driven musculoskeletal models are incorporated into the robot interaction controllers to account for differences of individuals and to provide personalised interaction/assistance capabilities. The authors in [Gribovskaya et al., 2011b] proposed an adaptation scheme for impedance parameters to enhance robot learning capabilities. In particular, Gaussian Mixture Models (GMM) were used to learn the task model, thus allowing the robot to anticipate the partner's intentions and to adapt its motion according to perceived interaction forces. The same concept was extended in [Kronander and Billard, 2014]. Similarly, the work in [Ragaglia et al., 2016] proposed a kinaesthetic teaching strategy (see also [Lee and Ott, 2011]), based on impedance control, which is able to achieve both high accuracy and repeatability of the motion of the robot, as it is hand-guided by the human operator. In [Mörtl et al., 2012], the authors proposed dynamic role exchange mechanisms and applied the approach to a collaborative transportation task. Here, the impedance control concept is used to simultaneously account for the load sharing problem, the motion control of the manipulated object and the minimisation of internal wrenches.

Alternative techniques aim at developing implicit interaction capabilities through real-time motion generation and trajectory re-planning methods. Visual perception is one of the commonly adopted sensory input in this area due to the possibility of tracking the human motion in real-time. In the work of Ebert et al. [Ebert and Henrich, 2002], a set of stationary $2 \mathrm{D}$ ceiling cameras were used to predict possible unsafe situations within a collaborative industrial work-cell. The system was able to predict possible collisions and to promptly command a stopping trajectory to the manipulator. Within a similar setting, in [Bascetta et al., 2011] the system is trained to predict long-term walking trajectories of the human worker in order to proactively trigger an appropriate interaction behaviour. The approach used GMM for modelling the data set of acquired trajectories and a Hidden Markov Model (HMM) for the online prediction phase.

A relatively recent line of research in the realm of humanrobot collaboration is established to generate natural human- like robot motions for an enhanced acceptability and compatibility to the human workspaces [Zanchettin et al., 2013b]. The applications of robotic learning techniques in this context has become indispensable. For instance, the authors in [Kim et al., 2006] exploited a motion capture database filled with human movements to generate human-like motion primitives. In a similar vein, authors in [Khatib et al., 2009] developed a musculoskeletal model to analytically describe the natural human motion and to minimise human fatigue. Calinon et al. [Calinon et al., 2010] proposed a probabilistic learning strategy to reproduce human-like motions through imitation. The approach is based on a combination of HMMs and Gaussian Mixture Regression (GMR) to learn and reproduce from a demonstrated set of data.

Given the variety of interfaces already discussed in Section 2 and the availability of different control strategies analysed so far, a natural field of new investigations is represented by multi-modal interaction: several modes among those discussed earlier in this Section are combined to form the interaction behaviour of the robotic manipulator. With the aim of mimicking the way humans interact with each other, the first combination of perception and control algorithms exploited the two mostly important senses: vision and touch (tactile perception). The first results on this topic were reported in [De Santis et al., 2007] where a control scheme, mainly targeting the enhancement of safety, has been proposed to account for both force and vision information. The authors in [Magrini et al., 2014] used visual information to understand the contact points between the human and the robotic manipulator. Another control architecture considering both visual and force information was presented in [Zanchettin and Rocco, 2015], where visual information was used to track an object while the human and the robotic manipulator are interacting by means of an impedance control scheme. In [Cherubini et al., 2013], the authors present a control scheme dealing with both visual perception and force information allowing the robot to infer human intentions during a collaborative screwing task. The different interaction modalities are sequenced based on the gathered sensor information. A multi-modal control strategy for a peer to peer collaborative carrying task has been presented in [Agravante et al., 2014b]. Here, the force and visual information are merged together to allow both safe and effective interaction. In particular, the former is mainly used to establish safety, while the latter is used to control the orientation of the transported object.

A more recent work in this area proposed a multi-modal interaction modality by implementing a hybrid impedance/force control in different Cartesian axes of the collaborative robot [Peternel et al., 2016c]. The force controller's objective was to establish and maintain the contact between a sawing tool and the environment. Instead, the impedance controller operated on a combined EMG-visual tracking interface to 
regulate the task stiffness and its frame in various phases of the collaborative task.

In general terms, the improved adaptability of the robotic platforms to unpredicted events has paved the way for the deployment of well-perfoming robots in complex interaction scenarios. However, the understanding the required levels of adaptivity from the two counterparts is still at its beginning, and a principled formalism is required to address the mutual human-robot adaptation requirements [Ikemoto et al., 2012].

\section{Stability and Transparency of the PHRC Systems}

When humans interact physically with robots, the robot control faces critical challenges in achieving performances while ensuring stability. Indeed, as well explained in [Buerger and Hogan, 2007], the stability of the human-robot system depends on the coupled dynamics. Interactions can alter both performance and stability of the two systems when coupled; hence, even if the two systems are stable in isolation, the coupled system may be unstable and not satisfy performance objectives. For example, a robotic manipulator may be very fast and precise in following a desired path along a known surface; a human may physically grab the robot to adapt the nominal trajectory to variability in the task: this interaction should always be "stable" regardless of the forces exerted by the human, gripping force, hand stiffness while grabbing and steering the robot, etc. Most controllers for such interacting systems regulate forces or dynamics behaviour of the robot at the interaction port (the location of the interaction, e.g., the end-effector). As mentioned before, a traditional control concept for achieving these performances is impedance control [Hogan, 1985], which consists in controlling the dynamic behaviour of the robot under the action of an external force, modelling the system as a spring-mass duo, with desired stiffness and damping.

Another desirable property of the robot control during interaction should be transparency [Lamy et al., 2009], e.g., in some applications, the robot should not oppose resistance to the force applied by the human operator when driving the robot, but it should rather move according to the intention of the human operator. This issue is particularly critical during co-manipulation, where an object is simultaneously held by the human and the robot. A similar performance is required in medical applications, such as assisted gesture guidance, but also in industrial contexts, such as assisted assembly.

Transparency is usually obtained by force feedback control. However, even transparency requires stability: a large bandwidth with high-force feedback gains is necessary to achieve a good transparency, but it has been shown in [Eppinger and Seering, 1987] that this condition may lead to instability if the environment is stiff (see in [Colgate and
Hogan, 1989] the concept of "contact instability"), or more generally if its impedance is unknown or varying.

Stability in interaction control is therefore linked to the control of mechanical impedance. In traditional robots, driven by stiff DC actuators, impedance is obtained by active force control: while many recent robots equipped with pervasive joint torque sensing and controlled with fast low-level loops (e.g., the KUKA iiwa) can achieve good response in safe impedance ranges, interaction control can be more challenging in other robots with proximal sensing (such as the iCub [Fumagalli et al., 2012] ). Indeed, time-delays in computing the active control and non-collocation (e.g., distance between sensors and actuation) can cause potential threats to the stability, reducing significantly for example the range of stable impedance values for interaction [Berret et al., 2011]. To increase the intrinsic compliance of the robot, there have been several robots equipped with elastic or flexible joints, and more recently with variable impedance actuators [Vanderborght et al., 2013b], which enable fast regulation of mechanical impedance at the joint level.

As well documented in [Buerger and Hogan, 2007], early works in interaction control assumed that impedance could be sculpted to achieve desired performances. However, achieving truly arbitrary impedance on any real robotic system is not possible, especially during interaction with humans, because the environment stiffness is unknown or varying, which may lead to coupled or contact instability [Colgate and Hogan, 1989]. This issue has been investigated using the concept of passivity: in [Colgate and Hogan, 1988], Colgate showed that when two stable systems with passive port functions are physically coupled, stability of the coupled system is guaranteed. However, passivity leads to very conservative gain tuning and constrain the apparent endpoint inertia to remain $50 \%$ higher than its real value, which prevents many co-manipulation applications with heavy robots [Lamy et al., 2009]. It is important to note here that in robots with joint torque sensing, the torque feedback can contribute to a substantial reduction of total inertia, while staying in a passivity framework [Albu-Schäffer et al., 2007].

Several studies showed that passivity may be too conservative and should be relaxed to increase performance during physical collaboration [Buerger and Hogan, 2006, Duchaine and Gosselin, 2008]. To relax the passivity constraint, Newman introduced the natural admittance control (NAC) [Newman, 1992], focused at reducing the apparent friction independent from inertia. Ficuciello et al. in [Ficuciello et al., 2014] proposed to use the robot's redundancy to ensure a decoupled apparent inertia at the end-effector thus increasing the range of stable impedance parameters. Buerger \& Hogan introduced the concept of "complementary stability" [Buerger and Hogan, 2007], which transforms the stability problem into a robust stability problem: by making the hypothesis that the human arm dynamics is unknown but 


\begin{tabular}{|c|c|c|c|c|}
\hline & Method & Pros & Cons & Use case examples in PHRC (robot control strategy) \\
\hline \multirow[t]{4}{*}{ Feed-forward } & Vision & $\begin{array}{l}\text { Suitable for motion plan- } \\
\text { ning and trajectory genera- } \\
\text { tion, Intuitive from the hu- } \\
\text { man standpoint }\end{array}$ & $\begin{array}{l}\text { Provides only a rough es- } \\
\text { timation of the contact dy- } \\
\text { namics }\end{array}$ & $\begin{array}{l}\text { [Morel et al., 1998] (impedance control), [Carlson and } \\
\text { Demiris, 2012] (shared control), [Agravante et al., 2014b] } \\
\text { (visual control), [Perzanowski et al., 1998, Sakita et al., } \\
\text { 2004, Li et al., 2005, Hawkins et al., 2013] (not concerned) }\end{array}$ \\
\hline & Auditory & $\begin{array}{l}\text { Intuitive from the human } \\
\text { standpoint }\end{array}$ & $\begin{array}{l}\text { Robots must run on a large } \\
\text { degree of autonomy to re- } \\
\text { spond to such high-level } \\
\text { commands }\end{array}$ & $\begin{array}{l}\text { [Medina et al., 2012b] (motion primitives), [Miyake and } \\
\text { Shimizu, 1994] (nonlinear oscillators), [Ivaldi et al., 2016] } \\
\text { (fixed joint impedance control) }\end{array}$ \\
\hline & Force/Pressure & $\begin{array}{l}\text { Accurate estimation of } \\
\text { the exchanged forces } \\
\text { and torques in contact, } \\
\text { Straightforward processing } \\
\text { and integration into the } \\
\text { control loop }\end{array}$ & $\begin{array}{l}\text { Interaction with uncertain } \\
\text { environment may add un- } \\
\text { desired components and } \\
\text { the robot's performance } \\
\text { may deteriorate }\end{array}$ & $\begin{array}{l}\text { [Kosuge and Kazamura, 1997a] (impedance control) [Al- } \\
\text { Jarrah and Zheng, 1997b] (compliant motion control), } \\
\text { [Ikeura and Inooka, 1995b, Tsumugiwa et al., 2002b, } \\
\text { Gribovskaya et al., 2011b] (variable impedance control) } \\
\text { [Agravante et al., 2014b] (visual control), [Duchaine and } \\
\text { Gosselin, 2009] (variable damping), [Peternel and Babič, } \\
\text { 2013](variable impedance control), [Palunko et al., 2014, } \\
\text { Donner and Buss, 2016b] (energy-based) }\end{array}$ \\
\hline & $\begin{array}{l}\text { Bio-signal } \\
\text { (EMG, } \\
\text { EEG, etc.) }\end{array}$ & $\begin{array}{l}\text { Adaptive and versatile, } \\
\text { Suitable for detecting hu- } \\
\text { man physical and cognitive } \\
\text { state variations }\end{array}$ & $\begin{array}{l}\text { Require advanced process- } \\
\text { ing techniques to extract } \\
\text { reliable control signals }\end{array}$ & $\begin{array}{l}\text { [Rani et al., 2004, Bell et al., 2008] (not concerned), } \\
\text { [Peternel et al., 2016b, Peternel et al., 2016c] (hybrid } \\
\text { force/impedance control) }\end{array}$ \\
\hline \multirow[t]{3}{*}{ Feed-back } & Vision & $\begin{array}{l}\text { Suitable for augmented re- } \\
\text { ality applications or remote } \\
\text { human-robot collaboration }\end{array}$ & $\begin{array}{l}\text { Not intuitive to indicate the } \\
\text { interaction forces in con- } \\
\text { tact, information overload- } \\
\text { ing in AR }\end{array}$ & $\begin{array}{l}\text { [Glassmire et al., 2004] (teleoperation), [Green et al., } \\
\text { 2009] (not concerned) }\end{array}$ \\
\hline & $\begin{array}{l}\text { Grounded } \\
\text { Force } \\
\text { Feedback }\end{array}$ & $\begin{array}{l}\text { Intuitive perception of the } \\
\text { interaction forces }\end{array}$ & $\begin{array}{l}\text { Usually not wear- } \\
\text { able/portable, May limit } \\
\text { the human natural work- } \\
\text { space, May deteriorate the } \\
\text { overall system's stability }\end{array}$ & $\begin{array}{l}\text { [Horiguchi et al., 2000] (teleoperation), [Yang et al., 2016] } \\
\text { (variable stiffness control), [Fernandez et al., 2001] (active } \\
\text { trajectory re-generation) }\end{array}$ \\
\hline & $\begin{array}{l}\text { Tactile } \\
\text { Feedback }\end{array}$ & $\begin{array}{l}\text { Lightweight and wearable } \\
\text { (e.g. mechano- and vibro- } \\
\text { tactile) }\end{array}$ & $\begin{array}{l}\text { Adequate training is re- } \\
\text { quired to use such sensory } \\
\text { substitution systems suc- } \\
\text { cessfully }\end{array}$ & [Ajoudani et al., 2014] (variable stiffness control) \\
\hline
\end{tabular}

Table 1: An overview of the HRC Interfaces and interaction modalities. Feed-forward interfaces are developed for robots to recognize human intentions and perceive the unknown environment. Feed-back interfaces provide additional input (through artificial systems) to improve human perception of the task and the environment. References include both uni- and multi-modal use of such interfaces to associate sensory input(s) to the artificial systems' (robot or the feedback device) functionalities.

reasonably bounded, the goal is to find the optimal tuning for a parameterized controller that is stable for the range of different environment dynamics. NAC and complementary stability were combined in [Lacevic and Rocco, 2011], but, as observed in [Dimeas and Aspragathos, 2016], their conservative gains were still hindering a smooth collaboration without efforts for the human operator. In [Dimeas and Aspragathos, 2016], the authors proposed online adaptation of an admittance controller gains on the basis of an instability index, computed in the frequency domain, that detects unstable behaviours of the human-robot coupled system by monitoring high-frequency oscillations in the force signal.

The main advantage of using admittance control schemes or optimal control schemes for adapting the gains and regulating the exchanged forces is that control theory provides rigorous frameworks and methods to prove the stability of the system and designing controls that are robust to perturbations. For interaction policies acquired or refined via machine learning techniques, such proofs are more difficult. If, for example, an interaction policy is learned from demonstration and refined through reinforcement learning (which is a common case in most robotics learning scenarios [Amor et al., 2009]), since the policy or its model are acquired on-line, and not known a priori, it is very difficult to provide stability proofs if not some conditions on the control bounds. In [Khansari-Zadeh and Billard, 2011], KhansariZadeh and Billard proposed a method, called SEDS, to learn stable parameterisations of dynamical systems used to reproduce robot movements learned by demonstrations. The method, exploiting Gaussian Mixture Models, ensures timeinvariance and appropriate reaction to perturbations thanks to a proof of global asymptotic stability at the target. However their approach was focused on stabilising movement trajectories, not specifically to stabilising impedance during interaction. In [Khansari-Zadeh et al., 2014], KhansariZadeh et al. proposed an extension of their framework that combines the problem of learning motion trajectories and regulating the impedance during interaction, ensuring global stability. They provided sufficient conditions to ensure global asymptotic stability for movements in free-space, and passivity during persistent contact with a passive environment.

Stability and transparency are primordial to achieve a safe physical interaction, particularly to allow the robot to follow human guidance. These properties are fundamental for the robot to optimize the collaboration introducing other features such as legibility (i.e., the property of generating legible motions that can be easily understood by the human partners) and anticipation (i.e., the property of predicting the human intention, the goal of the collaboration, and optimizing the robot control policy to take into account the human 
action, reducing the human effort or improving some shared performance criteria) [Stulp et al., 2015,Dragan et al., 2013]. Communication of intention in human-human physical interaction increases with mutual pratice, it is related to the arm impedance, to the continuous role adaptation between the partners [Mojtahedi et al., 2017] and it is also related to transparency [Jarrasse et al., 2008]: the same evidence holds for human-robot physical interaction [Dragan and Srinivasa, 2012, Jarrasse et al., 2013]. Transparency and stability are also crucial for optimizing the control in a way to provide assistance to the human while performing the task, as it is frequently done in robotic rehabilitation [Morasso et al., 2007].

\subsection{Lessons from human motor control}

The main problem in physical human-robot interaction is therefore tuning online the gains of the impedance or admittance control, for optimising performance, reducing the effort of the human operator ${ }^{4}$, and ensuring the stability.

Let us consider the simple case of the human arm interacting with a robotic manipulator at the end-effector. The main difficulty here for the robot is the accurate estimation of the varying environment, e.g., the end-point stiffness of the human arm [Ajoudani, 2016, Burdet et al., 2000]. To ensure stability and provide transparency, the impedance or admittance parameters should be tuned online according to the estimated human stiffness. A common way to estimate it, is via the grasping force at the interaction point, by dedicated force/torque sensors, even if in industrial applications there exists several cases where the human operator interacts directly with the payload, such as with lifting or assist devices. In [Lamy et al., 2009], the authors designed a handle for intuitive co-manipulation, where a 6-dof force/torque sensor was covered by a soft foam: when held delicately through a precision grip, the foam acts as a mechanical filter, and the system reflects low inertia; conversely, when held firmly through a power grasp, resulting from higher impedance in the arm because of muscle co-contraction [Napier, 1956], the squeezed foam does not filter, the system exhibits higher inertia, the gains are lowered and the system remains stable.

Human motor control actually provides several prior knowledge that can be exploited to adapt the impedance gains during physical interaction and more in general to optimize robot control [Ivaldi et al., 2012]. Dimeas and Aspragathos

\footnotetext{
4 The human effort can be identified in two stages: the physical and cognitive loads while learning a new collaborative task, and their amount on a regular basis after the human becomes an expert. This is important to note here that the CNS is capable of learning and adaptation to various tasks demands and disturbances, hence contributing to a reduction in the overall physical and cognitive loading while performing tasks with dynamic uncertainties [E. Burdet, 2001, Franklin et al., 2003]. Nevertheless, the (robot) counterpart's adaptive behaviour can affect such learning and adaptation processes in terms of time and performance.
}

observed in [Dimeas and Aspragathos, 2016] that the bandwidth of voluntary motion in humans is relatively low and below $2 \mathrm{~Hz}$ [de Vlugt et al., 2003], hence during physical interaction with the robot it is possible to discriminate the human operator's intent from the unstable motions thanks to frequency analysis. External oscillatory excitations of the arm up can be mitigated by the central nervous system (CNS) by changing the arm impedance: indeed, it has been shown by [E. Burdet, 2001] that the CNS can learn to control the magnitude, shape and orientation of the endpoint stiffness in a predictive way that is independent of the force needed to compensate for the imposed dynamics. Instead of simply co-contracting all the muscles, the CNS adopts the optimal strategy of increasing the impedance to be robust to external perturbations.

As discussed in [Yang et al., 2011], while in robots the common strategy to deal with perturbations and uncertainty is to design robust controllers that preserve stability, humans adapt the endpoint force and viscoelasticity to compensate for external forces, minimising error and effort while ensuring a constant stability margin. In fact, there is evidence that humans learn and adapt internal dynamic models of their own arm and of the environment during interaction [Shadmehr and Mussa-Ivaldi, 1994a], and these models are used for predicting muscle activations for planning movements. However, these feedforward signals alone are not sufficient in case of unstable dynamic environments: in several studies [Franklin et al., 2008, Franklin et al., 2007, Franklin et al., 2003], Franklin et al. reported evidence that the CNS regulates the arm compliance by co-contracting the muscles to cope with instability and feedback delays. More precisely, in [Franklin et al., 2007] the authors observed that the increase in stiffness was oriented along the direction of instability in the environment, confirming that the CNS is able to control the endpoint impedance of the limbs and selectively adapt it to the environment.

These studies support the idea that the CNS acts like an impedance controller at the level of the endpoint, ensuring stability, and reducing movement variability by increasing the impedance to reject disturbances. Upon these observations, in [Yang et al., 2011] the authors proposed a biomimetic controller with adaptive properties similar to those of humans. The controller increased feed-forward force and impedance in presence of large errors, whereas in absence of disturbance it decreased feed-forward force and impedance profiles while maintaining stability.

The aforementioned studies reported experimental evidence that the CNS can actually learn to control the interaction and adapt to different dynamics. In bimanual visuomotor tasks, even perturbed by deviating force fields, humans rapidly learn to control the interaction-forces by a combination of arm stiffness properties and direct force control [Squeri et al., 2010]. This property of the human mo- 
tor control system is exploited in robot assisted rehabilitation, where often assistance is provided by robotic devices via haptic signals [Morasso et al., 2007], to the point of changing the arm impedance and viscosity in impaired subjects [Piovesan et al., 2013].

More generally, the capability of humans to adapt/learn during physical interaction should be taken into account while designing a collaborative robotic system. The human can rapidly learn how to proficiently interact with the robot in visuo-motor tasks such as guiding and tracking, and can "adapt" to novel dynamics. A continuous interaction with the robot can make them become "expert" in the collaboration, at the price of a (small) cognitive load on the task. Therefore, an interesting research question arises: does the robot need to continuously adapt to the human partner, forcing him/her to re-adapt (thus increasing the task load, both cognitively and physically), or should it keep its control policy to exploit the fast learning capabilities of the human to (simply) improve the collaboration?

The question is very relevant as a continuous interaction will make the human "expert", however this holds if the robot does not change its policy, forcing the human to readapt. There may be cases where a continuous re-adaptation from both sides would make sense and lead to more efficient collaboration, for example when the robot has to collaborate with a variety of different partners and could improve its skills by continuous learning from the different partners. However, to the authors' knowledge there are not yet results showing the benefit of long-term multi-partner adaptation on collaborative tasks between robots and humans in real world applications.

\section{Benchmarking and Relevant Use Cases}

Over the last decade, several research groups aimed at evaluating the quality of human-robot interaction and collaboration by examining the acceptability of the framework by human volunteers. For example, authors in [Kahn et al., 2006] and [Feil-Seifer et al., 2007] proposed several benchmarking methods based on psychological assessments. In particular, a common approach, at the time, was to divide the assessment into three categories: one based on the robot (addressing safety and scalability), another based on social interaction (autonomy, imitation, privacy, understanding of specific domain, social success), and the latter based on the impact of the assistive technology on the users.

Other contributions additionally addressed the anthropomorphism of robotic agents, by confirming or invalidating the well-known concept of the Uncanny Valley [Hegel et al., 2008, Hwang et al., 2013]. The first studies were mainly focused on subjective responses, like questionnaires. Later on, based on the pioneering work of Kulic and Croft (see [Kulic and Croft, 2007]) the focus was moved towards a more objective evaluation, and no longer focused on pure appearance of robots, but rather on their motion capabilities (see also [Zanchettin et al., 2013a, Oztop et al., 2005, Kilner et al., 2003]). Recently, the authors in [Bestick et al., 2015] provided some objective insights on the benefits of a personalised robot behaviour on human physiological measurements. As of today, however, no significant evaluation procedures specifically targeting physical interaction is reported in literature.

When it comes to validating research results within effective and significant demonstrations, several benchmarking applications are introduced. Collaborative transportation of bulky and/or heavy objects is one the most common candidates to test collaboration modalities and interfaces. In most of the cases, the object to be manipulated is a rigid, non-articulated one, like a box or a planar surface, as reported in [Al-Jarrah and Zheng, 1997b, De Schutter et al., 2007, Wojtara et al., 2009, Mörtl et al., 2012, Agravante et al., 2014b]. The most challenging aspects of this demonstration are related to the task definition (both the human and the robot have to agree on the direction). Particularly interesting, from an ergonomic point of view, the focus is on how the problem of sharing the carrying the effort is solved. For example in [Rozo et al., 2013] a learning strategy is combined with an impedance controller to perform a collaborative assembly task on a wooden table. In particular, while the robot is holding the table, the human can easily mount the legs as the robot automatically tunes its stiffness parameters to facilitate the completion of the task. The authors in [Kim et al., 2017] proposed a technique to monitor the overloading joint torque profiles (due to the external load) of the human partner for ergonomic and comfortable execution of heavy co-manipulation tasks. The authors of [Edsinger and Kemp, 2007] presented an object handover task between a robot and a human. The manipulator stiffness is adapted to accommodate for the physical interaction with the object and to ultimately accomplish the task.

Apart from the obvious domestic applications, this collaborative task has an immediate application in construction sites, as described in [Lee et al., 2007] where a miniexcavator was developed and used for the installation of curtain walls. Relevant applications of physical human-robot interaction are also reported in the healthcare field. Apart from rehabilitation robotics, which is not the target of this review paper, robotic assistance is the main relevant application. For example, in [Chuy et al., 2006] a collaborative mobile robot is developed in order to assist people to walk, despite physical inabilities. In [Ulrich and Borenstein, 2001] a haptic guidance cane is developed for the assistance of visually impaired people. A similar application scenario is also described in [Wakita et al., 2013].

In the field of industrial robotics, many manufacturing applications are also reported in literature, ranging from load- 
ing and transporting heavy wheels [Levratti et al., 2016], collaborative assembly of a homokinetic mechanical joint [Cherubini et al., 2013, Cherubini et al., 2016] and cellular phones [Tan et al., 2009]. Another application within this field was presented in [Erden and Billard, 2014]. Here, an impedance controlled robot is used both to measure and learn the end-point impedance of expert welders for subsequent autonomous execution as well as for the training of non-skilled personnel. More recently, authors in [Peternel et al., 2016c, Peternel et al., 2016b] performed a collaborative sawing task as an illustrative example of dynamic industrial tasks with environmental uncertainties.

Some other developments are focused on collaborative aspects in manipulating non rigid or articulated objects [Colomé et al., 2015]. For example, in [Kosuge et al., 1998] the robot was commanded to deform a flexible metal sheet and support its payload so that the human could easily handle it. Similarly, in [Kruse et al., 2015] a collaborative robots was employed to help the human to fold a tablecloth. As for other examples, [Maeda et al., 2001] a collaborative rope turning was presented, while in [Donner and Buss, 2016a] the human and a robotic arm were collaboratively manipulating pendulum-like objects in order to established a limit cycle with the desired amount of energy. Deformable materials are also of concern within domestic service robotics.

Finally, a recent trend in collaborative robotics research is devoted to the design of robots that take into account the ergonomics requirements typical of industrial applications [Maurice et al., 2017]. This field of research is not only promising but very important for the design of ergonomically compatible robots, as well as for the design of robot controls that optimize human ergonomics performances.

\section{Discussions and outlook}

The enhanced physical dexterity of the new generation of robotic platforms has paved the way towards their integration into robotics enabled service and care applications. Among various interaction scenarios, the exploitation of robots in collaborative settings has created a very important and high impact initiative. While HRC's significant economic impact on industry is expected at large, it will also serve to maximise the social impact by maintaining the sense of purpose of the involved people in the work process.

In this paper, we provided an overview of the current state-of-the-art in human-robot collaboration. Although the hardware components of such systems are among the most crucial for a successful market entry, this review paper was dedicated to other important aspects, including the intermediate interfaces for improved human and robot perception; robot control modalities to perform on these channels (interaction modalities); the control performances (stability and transparency); and potential use cases (benchmarking).
The review of previous work on feed-forward (allowing the robot to perceive the human) and feed-back (complementing human perception of the task and the environment) communication channels indicated that the implementation of multi-modal human-robot interfaces presents a big advantage in addressing complex interaction scenarios. Nevertheless, it is likely to be accompanied by an increase on the software complexity and the human cognitive burden (for operating such modalities). Hence, an appropriate trade-off between the complexity and completeness of human sensorimotor behaviour and intention modelling must be defined. On the other hand, a considerable effort must be made to understand the required level of human awareness about the robot and environment states through artificial communication channels. In particular, despite the availability of several technologies, e.g. force feedback, augmented reality, etc., the amount of information (and its level of detail) the robot should communicate to the human is still an open research topic.

It was also indicated that the implementation of adaptive control methodologies such as impedance, force, admittance or hybrid approaches will not only enhance the robot adaptivity to the human and the environment, it may also lead to a reduced level of task-related pre-programming. Nevertheless, the application of such control concepts in human-robot collaboration still appears in a premature state and must be evaluated in cross-application HRC scenarios. In this direction, a principled human-robot mutual adaptation formalism is required to understand the levels of adaptivity from the two counterparts, and to address some questions arising on this topic: e.g., should the robot adaptation be generic or user-specific? As the robot eventually has some degree of controllability on the human companion, should this be still conceived as ethical?

Another very important aspect to be considered here is the underlying human and robot safety, so that well-performing robots can be successfully and seamlessly integrated in humancollaborative settings. Although this topic was not directly addressed in this review (due to the existence of a dense body if literature to discuss this aspect [Haddadin et al., 2009, De Santis et al., 2008, Alami et al., 2006]), it out to be mentioned that a great deal of effort must be directed towards ensuring safety for collaborating humans (to avoid injuries and accidents) and robots (to avoid unacceptable economic losses).

A list of potential applications and relevant use cases ranging from domestic to industrial environments was provided. A quick review of the state-of-the-art suggests that the research community's attention is being extended from static tasks within structured environments to more dynamic collaborative scenarios that are subject to un-modelled dynamics and uncertainties, to facilitate a large-scale integration of robotic systems in several real-world scenarios. 
Finally, several benchmarking examples were reported, with the underlying analysis mainly focusing on the acceptability aspects, either from an explicit (e.g. using questionaries) or implicit (using physiological data) perspective. A natural problem arising from human-robot collaboration is to evaluate how the overall system behaviour, composed by the robot and the human, with respect to a certain set of performance indices. Such indices can be task-specific (a common approach in industry), however, this may limit the cross-application comparability of the HRC frameworks.

To conclude, this review paper was intended to give an updated overview of the state-of-the-art and recent research trends in human-robot collaboration. Despite the fast-growing interest in the real-world applications of the HRC, several challenges are still awaiting to be solved.

\section{Acknowledgements}

The authors would like to thank and remember Fabrizio Flacco for his spirit, contributions, and enthusiasm for writing this review paper. We will keep his memories alive in our hearts.

This work is supported in part by the EU FP7-ICT projects WALKMAN (no. 611832) and CoDyCo (no. 600716); in part by the H2020 Projects SoftPro (no. 688857) and AnDy (no.731540).

\section{References}

Adams et al., 1996. Adams, J., Bajcsy, R., Košecká, J., Kumar, V., Mintz, M., Mandelbaum, R., Wang, C.-C., Yamamoto, Y., and Yun, X. (1996). Cooperative material handling by human and robotic agents: Module development and system synthesis. Expert Systems with Applications, 11(2):89-97.

Agravante et al., 2014a. Agravante, D., Cherubini, A., Bussy, A., Gergondet, P., and Kheddar, A. (2014a). Collaborative human-humanoid carrying using vision and haptic sensing. In Robotics and Automation (ICRA), 2014 IEEE Intl. Conf. on, pages 607-612.

Agravante et al., 2014b. Agravante, D. J., Cherubini, A., Bussy, A., Gergondet, P., and Kheddar, A. (2014b). Collaborative humanhumanoid carrying using vision and haptic sensing. In 2014 IEEE International Conference on Robotics and Automation (ICRA), pages 607-612. IEEE.

Ajoudani, 2016. Ajoudani, A. (2016). Transferring Human Impedance Regulation Skills to Robots. Springer.

Ajoudani et al., 2014. Ajoudani, A., Godfrey, S. B., Bianchi, M., Catalano, M. G., Grioli, G., Tsagarakis, N., and Bicchi, A. (2014). Exploring teleimpedance and tactile feedback for intuitive control of the pisa/iit softhand. IEEE transactions on haptics, 7(2):203-215.

Al-Jarrah and Zheng, 1997a. Al-Jarrah, O. and Zheng, Y. (1997a). Arm-manipulator coordination for load sharing using reflexive motion control. In Robotics and Automation (ICRA), 1997 IEEE Intl. Conf. on, volume 3, pages 2326-2331.

Al-Jarrah and Zheng, 1997b. Al-Jarrah, O. M. and Zheng, Y. F. (1997b). Arm-manipulator coordination for load sharing using reflexive motion control. In Robotics and Automation, 1997. Proceedings., 1997 IEEE International Conference on, volume 3, pages 2326-2331. IEEE.
Alami et al., 2006. Alami, R., Albu-Schäffer, A., Bicchi, A., Bischoff, R., Chatila, R., De Luca, A., De Santis, A., Giralt, G., Guiochet, J., Hirzinger, G., et al. (2006). Safe and dependable physical humanrobot interaction in anthropic domains: State of the art and challenges. In 2006 IEEE/RSJ International Conference on Intelligent Robots and Systems, pages 1-16. IEEE.

Albu-Schäffer et al., 2007. Albu-Schäffer, A., Haddadin, S., Ott, C., Stemmer, A., Wimböck, T., and Hirzinger, G. (2007). The DLR lightweight robot: design and control concepts for robots in human environments. Industrial Robot: An International Journal, 34(5):376385.

Albu-Schäffer et al., 2007. Albu-Schäffer, A., Ott, C., and Hirzinger, G. (2007). A unified passivity-based control framework for position, torque and impedance control of flexible joint robots. The International Journal of Robotics Research, 26(1):23-39.

Amor et al., 2009. Amor, H. B., Berger, E., Vogt, D., and Jung, B. (2009). Kinesthetic bootstrapping: Teaching motor skills to humanoid robots through physical interaction. In Annual Conference on Artificial Intelligence, pages 492-499. Springer.

Argall and Billard, 2010. Argall, B. D. and Billard, A. G. (2010). A survey of tactile human-robot interactions. Robotics and $\mathrm{Au}$ tonomous Systems, 58(10):1159-1176.

Bascetta et al., 2011. Bascetta, L., Ferretti, G., Rocco, P., Ardö, H., Bruyninckx, H., Demeester, E., and Di Lello, E. (2011). Towards safe human-robot interaction in robotic cells: an approach based on visual tracking and intention estimation. In 2011 IEEE/RSJ International Conference on Intelligent Robots and Systems, pages 29712978. IEEE.

Bauer et al., 2008. Bauer, A., Wollherr, D., and Buss, M. (2008). Human-robot collaboration: a survey. International Journal of $\mathrm{Hu}$ manoid Robotics, 5(01):47-66.

Bell et al., 2008. Bell, C. J., Shenoy, P., Chalodhorn, R., and Rao, R. P. (2008). Control of a humanoid robot by a noninvasive braincomputer interface in humans. Journal of neural engineering, $5(2): 214$.

Berret et al., 2011. Berret, B., Ivaldi, S., Nori, F., and Sandini, G. (2011). Stochastic optimal control with variable impedance manipulators in presence of uncertainties and delayed feedback. In Proceedings of the 2011 IEEE/RSJ International Conference on Intelligent Robots and Systems (IROS), pages 4354-4359.

Bestick et al., 2015. Bestick, A. M., Burden, S. A., Willits, G., Naikal, N., Sastry, S. S., and Bajcsy, R. (2015). Personalized kinematics for human-robot collaborative manipulation. In Intelligent Robots and Systems (IROS), 2015 IEEE/RSJ International Conference on, pages 1037-1044. IEEE.

Böhme et al., 2003. Böhme, H.-J., Wilhelm, T., Key, J., Schauer, C., Schröter, C., Groß, H.-M., and Hempel, T. (2003). An approach to multi-modal human-machine interaction for intelligent service robots. Robotics and Autonomous Systems, 44(1):83-96.

Buerger and Hogan, 2006. Buerger, S. P. and Hogan, N. (2006). Relaxing passivity for human-robot interaction. In 2006 IEEE/RSJ International Conference on Intelligent Robots and Systems, pages 4570-4575.

Buerger and Hogan, 2007. Buerger, S. P. and Hogan, N. (2007). Complementary stability and loop shaping for improved human-robot interaction. IEEE Transactions on Robotics, 23(2):232-244.

Burdet et al., 2000. Burdet, E., Osu, R., Franklin, D., Yoshioka, T., Milner, T., and Kawato, M. (2000). A method for measuring endpoint stiffness during multi-joint arm movements. Journal of biomechanics, 33(12):1705-1709.

Calinon et al., 2010. Calinon, S., D'halluin, F., Sauser, E. L., Caldwell, D. G., and Billard, A. G. (2010). Learning and reproduction of gestures by imitation. IEEE Robotics \& Automation Magazine, 17(2):44-54.

Carlson and Demiris, 2012. Carlson, T. and Demiris, Y. (2012). Collaborative control for a robotic wheelchair: evaluation of performance, attention, and workload. IEEE Transactions on Systems. Man, and Cybernetics, Part B (Cybernetics), 42(3):876-888. 
Castellini et al., 2014. Castellini, C., Artemiadis, P., Wininger, M., Ajoudani, A., Alimusaj, M., Bicchi, A., Caputo, B., Craelius, W., Dosen, S., Englehart, K., et al. (2014). Proceedings of the first workshop on peripheral machine interfaces: Going beyond traditional surface electromyography. Frontiers in neurorobotics, 8:22.

Cherubini et al., 2016. Cherubini, A., Passama, R., Crosnier, A., Lasnier, A., and Fraisse, P. (2016). Collaborative manufacturing with physical human-robot interaction. Robotics and ComputerIntegrated Manufacturing, 40:1-13.

Cherubini et al., 2013. Cherubini, A., Passama, R., Meline, A., Crosnier, A., and Fraisse, P. (2013). Multimodal control for human-robot cooperation. In 2013 IEEE/RSJ International Conference on Intelligent Robots and Systems, pages 2202-2207. IEEE.

Chuy et al., 2006. Chuy, O., Hirata, Y., and Kosuge, K. (2006). A new control approach for a robotic walking support system in adapting user characteristics. IEEE Transactions on Systems, Man, and Cybernetics, Part C (Applications and Reviews), 36(6):725-733.

Colgate and Hogan, 1989. Colgate, E. and Hogan, N. (1989). An analysis of contact instability in terms of passive physical equivalents. In Proceedings, 1989 International Conference on Robotics and Automation, pages 404-409.

Colgate and Hogan, 1988. Colgate, J. E. and Hogan, N. (1988). Robust control of dynamically interacting systems. International Journal of Control, 48(1):65-88.

Colomé et al., 2015. Colomé, A., Planells, A., and Torras, C. (2015). A friction-model-based framework for reinforcement learning of robotic tasks in non-rigid environments. In Robotics and Automation (ICRA), 2015 IEEE International Conference on, pages 5649-5654. IEEE.

Corrales et al., 2008. Corrales, J. A., Candelas, F., and Torres, F. (2008). Hybrid tracking of human operators using imu/uwb data fusion by a kalman filter. In Human-Robot Interaction (HRI), 2008 3rd ACM/IEEE International Conference on, pages 193-200. IEEE.

De Santis et al., 2007. De Santis, A., Lippiello, V., Siciliano, B., and Villani, L. (2007). Human-robot interaction control using force and vision. In Advances in Control Theory and Applications, pages 5170. Springer.

De Santis et al., 2008. De Santis, A., Siciliano, B., De Luca, A., and Bicchi, A. (2008). An atlas of physical human-robot interaction. Mechanism and Machine Theory, 43(3):253-270.

De Schutter et al., 2007. De Schutter, J., De Laet, T., Rutgeerts, J., Decré, W., Smits, R., Aertbeliën, E., Claes, K., and Bruyninckx, H. (2007). Constraint-based task specification and estimation for sensor-based robot systems in the presence of geometric uncertainty. The International Journal of Robotics Research, 26(5):433-455.

de Vlugt et al., 2003. de Vlugt, E., Schouten, A. C., van der Helm, F. C., Teerhuis, P. C., and Brouwn, G. G. (2003). A force-controlled planar haptic device for movement control analysis of the human arm. Journal of Neuroscience Methods, 129(2):151 - 168.

Dimeas and Aspragathos, 2016. Dimeas, F. and Aspragathos, N. (2016). Online stability in human-robot cooperation with admittance control. IEEE Transactions on Haptics, 9(2):267-278.

Donner and Buss, 2016a. Donner, P. and Buss, M. (2016a). Cooperative swinging of complex pendulum-like objects: Experimental evaluation. IEEE Transactions on Robotics, 32(3):744-753.

Donner and Buss, 2016b. Donner, P. and Buss, M. (2016b). Cooperative swinging of complex pendulum-like objects: Experimental evaluation. IEEE Transactions on Robotics, 32(3):744-753.

Dragan et al., 2013. Dragan, A., Lee, K., and Srinivasa, S. (2013). Legibility and predictability of robot motion. In Human-Robot Interaction, Pittsburgh, PA.

Dragan and Srinivasa, 2012. Dragan, A. and Srinivasa, S. (2012). Formalizing assistive teleoperation. In Robotics: Science and Systems, Pittsburgh, PA.

Duchaine and Gosselin, 2007. Duchaine, V. and Gosselin, C. (2007). General model of human-robot cooperation using a novel velocity based variable impedance control. In Eurohaptics Conference and
Symposium on Haptic Interfaces for Virtual Environment and Teleoperator Systems, 2rd Joint, pages 446-451.

Duchaine and Gosselin, 2009. Duchaine, V. and Gosselin, C. (2009). Safe, stable and intuitive control for physical human-robot interaction. In Robotics and Automation, 2009. ICRA'09. IEEE International Conference on, pages 3383-3388. IEEE.

Duchaine and Gosselin, 2008. Duchaine, V. and Gosselin, C. M. (2008). Investigation of human-robot interaction stability using lyapunov theory. In Robotics and Automation, 2008. ICRA 2008. IEEE International Conference on, pages 2189-2194. IEEE.

Dumora et al., 2012. Dumora, J., Geffard, F., Bidard, C., Brouillet, T., and Fraisse, P. (2012). Experimental study on haptic communication of a human in a shared human-robot collaborative task. In IEEE/RSJ International Conference on Intelligent Robots and Systems (IROS), pages 5137-5144.

E. Burdet, 2001. E. Burdet, R. Osu, D. W. F. T. E. M. M. K. (2001). The central nervous system stabilizes unstable dynamics by learning optimal impedance. Nature, pages 446-449.

Ebert and Henrich, 2002. Ebert, D. M. and Henrich, D. D. (2002). Safe human-robot-cooperation: Image-based collision detection for industrial robots. In Intelligent Robots and Systems, 2002. IEEE/RSJ International Conference On, volume 2, pages 1826-1831. IEEE.

Edsinger and Kemp, 2007. Edsinger, A. and Kemp, C. C. (2007). Human-robot interaction for cooperative manipulation: Handing objects to one another. In RO-MAN 2007-The 16th IEEE International Symposium on Robot and Human Interactive Communication, pages 1167-1172. IEEE.

Eppinger and Seering, 1987. Eppinger, S. and Seering, W. (1987). Understanding bandwidth limitations in robot force control. In Proceedings. 1987 IEEE International Conference on Robotics and Automation, volume 4, pages 904-909.

Erden and Billard, 2014. Erden, M. S. and Billard, A. (2014). Endpoint impedance measurements at human hand during interactive manual welding with robot. In 2014 IEEE International Conference on Robotics and Automation (ICRA), pages 126-133. IEEE.

Evrard et al., 2009. Evrard, P., Gribovskaya, E., Calinon, S., Billard, A., and Kheddar, A. (2009). Teaching physical collaborative tasks: object-lifting case study with a humanoid. In IEEE-RAS Intl. Conf. on Humanoid Robots, pages 399-404.

Evrard and Kheddar, 2009. Evrard, P. and Kheddar, A. (2009). Homotopy switching model for dyad haptic interaction in physical collaborative tasks. In Eurohaptics Conference and Symposium on Haptic Interfaces for Virtual Environment and Teleoperator Systems, 3rd Joint, pages 45-50.

Farina et al., 2014. Farina, D., Jiang, N., Rehbaum, H., Holobar, A., Graimann, B., Dietl, H., and Aszmann, O. C. (2014). The extraction of neural information from the surface EMG for the control of upperlimb prostheses: Emerging avenues and challenges. IEEE Transactions on Neural Systems and Rehabilitation Engineering, 22(4):797809.

Farry et al., 1996. Farry, K., Walker, I., and Baraniuk, R. (1996). Myoelectric teleoperation of a complex robotic hand. Robotics and $\mathrm{Au}$ tomation, IEEE Transactions on, 12(5):775-788.

Feil-Seifer et al., 2007. Feil-Seifer, D., Skinner, K., and Matarić, M. J. (2007). Benchmarks for evaluating socially assistive robotics. Interaction Studies, 8(3):423-439.

Fernandez et al., 2001. Fernandez, V., Balaguer, C., Blanco, D., and Salichs, M. A. (2001). Active human-mobile manipulator cooperation through intention recognition. In Robotics and Automation, 2001. Proceedings 2001 ICRA. IEEE International Conference on, volume 3, pages 2668-2673. IEEE.

Ficuciello et al., 2014. Ficuciello, F., Romano, A., Villani, L., and Siciliano, B. (2014). Cartesian impedance control of redundant manipulators for human-robot co-manipulation. In 2014 IEEE/RSJ International Conference on Intelligent Robots and Systems, pages 21202125. IEEE. 
Fleischer and Hommel, 2008. Fleischer, C. and Hommel, G. (2008). A human-exoskeleton interface utilizing electromyography. Trans. Rob., 24(4):872-882.

Fong et al., 2003. Fong, T., Nourbakhsh, I., and Dautenhahn, K. (2003). A survey of socially interactive robots. Robotics and autonomous systems, 42(3):143-166.

Franklin et al., 2008. Franklin, D. W., Burdet, E., Tee, K. P., Osu, R., Chew, C.-M., Milner, T. E., and Kawato, M. (2008). Cns learns stable, accurate, and efficient movements using a simple algorithm. The Journal of Neuroscience, 28(44):11165-11173.

Franklin et al., 2007. Franklin, D. W., Liaw, G., Milner, T. E., Osu, R., Burdet, E., and Kawato, M. (2007). Endpoint stiffness of the arm is directionally tuned to instability in the environment. The Journal of neuroscience, 27(29):7705-7716.

Franklin et al., 2003. Franklin, D. W., Osu, R., Burdet, E., Kawato, M., and Milner, T. E. (2003). Adaptation to stable and unstable dynamics achieved by combined impedance control and inverse dynamics model. Journal of neurophysiology, 90(5):3270-3282.

Freedy et al., 2007. Freedy, A., DeVisser, E., Weltman, G., and Coeyman, N. (2007). Measurement of trust in human-robot collaboration. In Collaborative Technologies and Systems, 2007. CTS 2007. International Symposium on, pages 106-114. IEEE.

Fumagalli et al., 2012. Fumagalli, M., Ivaldi, S., Randazzo, M., Natale, L., Metta, G., Sandini, G., and Nori, F. (2012). Force feedback exploiting tactile and proximal force/torque sensing. Autonomous Robots, 33(4):381-398.

Gams et al., 2014. Gams, A., Nemec, B., Ijspeert, A., and Ude, A. (2014). Coupling movement primitives: Interaction with the environment and bimanual tasks. Robotics, IEEE Transactions on, 30(4):816-830.

Gijsberts et al., 2014. Gijsberts, A., Bohra, R., Sierra González, D., Werner, A., Nowak, M., Caputo, B., Roa, M. A., and Castellini, C. (2014). Stable myoelectric control of a hand prosthesis using nonlinear incremental learning. Frontiers in neurorobotics, 8:8.

Glassmire et al., 2004. Glassmire, J., O'Malley, M., Bluethmann, W., and Ambrose, R. (2004). Cooperative manipulation between humans and teleoperated agents. In Haptic Interfaces for Virtual Environment and Teleoperator Systems, 2004. HAPTICS'04. Proceedings. 12th International Symposium on, pages 114-120. IEEE.

Godfrey et al., 2013. Godfrey, S., Ajoudani, A., Catalano, M., Grioli, G., and Bicchi, A. (2013). A synergy-driven approach to a myoelectric hand. In Rehabilitation Robotics (ICORR), 2013 IEEE International Conference on, pages 1-6. IEEE.

Gordon et al., 1993. Gordon, A. M., Westling, G., Cole, K. J., and Johansson, R. S. (1993). Memory representations underlying motor commands used during manipulation of common and novel objects. Journal of Neurophysiology, 69(6):1789-1796.

Green et al., 2008. Green, S. A., Billinghurst, M., Chen, X., and Chase, J. G. (2008). Human-robot collaboration: A literature review and augmented reality approach in design. International journal of advanced robotic systems, 5(1): 1 .

Green et al., 2009. Green, S. A., Chase, J. G., Chen, X., and Billinghurst, M. (2009). Evaluating the augmented reality humanrobot collaboration system. International journal of intelligent systems technologies and applications, 8(1-4):130-143.

Gribovskaya et al., 2011a. Gribovskaya, E., Kheddar, A., and Billard, A. (2011a). Motion learning and adaptive impedance for robot control during physical interaction with humans. In Robotics and $A u$ tomation (ICRA), 2011 IEEE Intl. Conf. on, pages 4326-4332.

Gribovskaya et al., 2011b. Gribovskaya, E., Kheddar, A., and Billard, A. (2011b). Motion learning and adaptive impedance for robot control during physical interaction with humans. In Robotics and $\mathrm{Au}$ tomation (ICRA), 2011 IEEE International Conference on, pages 4326-4332. IEEE.

Haddadin et al., 2009. Haddadin, S., Albu-Schäffer, A., and Hirzinger, G. (2009). Requirements for safe robots: Measurements, analysis and new insights. The International Journal of Robotics Research, 28(11-12):1507-1527.
Hawkins et al., 2013. Hawkins, K. P., Vo, N., Bansal, S., and Bobick, A. F. (2013). Probabilistic human action prediction and waitsensitive planning for responsive human-robot collaboration. In 2013 13th IEEE-RAS International Conference on Humanoid Robots (Humanoids), pages 499-506. IEEE.

Hegel et al., 2008. Hegel, F., Krach, S., Kircher, T., Wrede, B., and Sagerer, G. (2008). Understanding social robots: A user study on anthropomorphism. In RO-MAN 2008-The 17th IEEE International Symposium on Robot and Human Interactive Communication, pages 574-579. IEEE.

Hogan, 1985. Hogan, N. (1985). Impedance control: An approach to manipulation: Part iiimplementation. Journal of dynamic systems, measurement, and control, 107(1):8-16.

Horiguchi et al., 2000. Horiguchi, Y., Sawaragi, T., and Akashi, G. (2000). Naturalistic human-robot collaboration based upon mixedinitiative interactions in teleoperating environment. In Systems, Man, and Cybernetics, 2000 IEEE International Conference on, volume 2, pages 876-881. IEEE.

Hwang et al., 2013. Hwang, J., Park, T., and Hwang, W. (2013). The effects of overall robot shape on the emotions invoked in users and the perceived personalities of robot. Applied ergonomics, 44(3):459471.

Ikemoto et al., 2012. Ikemoto, S., Ben Amor, H., Minato, T., Jung, B., and Ishiguro, H. (2012). Physical human-robot interaction: Mutual learning and adaptation. Robotics Automation Magazine, IEEE, 19(4): $24-35$.

Ikeura and Inooka, 1995a. Ikeura, R. and Inooka, H. (1995a). Variable impedance control of a robot for cooperation with a human. In $R o$ botics and Automation (ICRA), 1995 IEEE Intl. Conf. on, volume 3, pages $3097-3102$.

Ikeura and Inooka, 1995b. Ikeura, R. and Inooka, H. (1995b). Variable impedance control of a robot for cooperation with a human. In Robotics and Automation, 1995. Proceedings., 1995 IEEE International Conference on, volume 3, pages 3097-3102. IEEE.

Ivaldi et al., 2014. Ivaldi, S., Anzalone, S., Rousseau, W., Sigaud, O., and Chetouani, M. (2014). Robot initiative in a team learning task increases the rhythm of interaction but not the perceived engagement. Frontiers in Neurorobotics, 8:5.

Ivaldi et al., 2016. Ivaldi, S., Lefort, S., Peters, J., Chetouani, M., Provasi, J., and Zibetti, E. (2016). Towards engagement models that consider individual factors in hri: on the relation of extroversion and negative attitude towards robots to gaze and speech during a humanrobot assembly task. Int. Journal of Social Robotics.

Ivaldi et al., 2012. Ivaldi, S., Sigaud, O., Berret, B., and Nori, F. (2012). From humans to humanoids: The optimal control framework. Paladyn, 3(2):75-91.

Jarrasse et al., 2008. Jarrasse, N., Paik, J., Pasqui, V., and Morel, G. (2008). How can human motion prediction increase transparency? In Proceedings of the IEEE International Conference on Robotics and Automation (ICRA'08), pages 2134-2139, Pasadena, California, US.

Jarrasse et al., 2013. Jarrasse, N., Sanguinetti, V., and Burdet, E. (2013). Slaves no longer: review on role assignment for human-robot joint motor action. Adaptive Behavior ( $S A G E$ ).

Jiang et al., 2009. Jiang, N., Englehart, K., and Parker, P. (2009). Extracting simultaneous and proportional neural control information for multiple-dof prostheses from the surface electromyographic signal. Biomedical Engineering, IEEE Transactions on, 56(4):1070-1080.

Johansson, 1998. Johansson, R. S. (1998). Sensory input and control of grip. Sensory guidance of movement, pages 45-59.

Kahn et al., 2006. Kahn, P. H., Ishiguro, H., Friedman, B., and Kanda, T. (2006). What is a human?-toward psychological benchmarks in the field of human-robot interaction. In Robot and Human Interactive Communication, 2006. ROMAN 2006. The 15th IEEE International Symposium on, pages 364-371. IEEE.

Kaneko et al., 2008. Kaneko, K., Harada, K., Kanehiro, F., Miyamori, G., and Akachi, K. (2008). Humanoid robot hrp-3. In 2008 IEEE/RSJ 
International Conference on Intelligent Robots and Systems, pages 2471-2478. IEEE.

Khansari-Zadeh and Billard, 2011. Khansari-Zadeh, S. and Billard, A. (2011). Learning stable nonlinear dynamical systems with gaussian mixture models. IEEE Transactions on Robotics, 27(5):943-957.

Khansari-Zadeh et al., 2014. Khansari-Zadeh, S., Kronander, K., and Billard, A. (2014). Modeling robot discrete movements with statevarying stiffness and damping: A framework for integrated motion generation and impedance control. In RSS.

Khatib et al., 2009. Khatib, O., Demircan, E., De Sapio, V., Sentis, L., Besier, T., and Delp, S. (2009). Robotics-based synthesis of human motion. Journal of Physiology-Paris, 103(3):211-219.

Kilner et al., 2003. Kilner, J. M., Paulignan, Y., and Blakemore, S.-J. (2003). An interference effect of observed biological movement on action. Current biology, 13(6):522-525.

Kim et al., 2006. Kim, S., Kim, C., and Park, J. H. (2006). Humanlike arm motion generation for humanoid robots using motion capture database. In 2006 IEEE/RSJ International Conference on Intelligent Robots and Systems, pages 3486-3491. IEEE.

Kim et al., 2017. Kim, W., Lee, J., Tsagarakis, N., and Ajoudani, A. (2017). A real-time and reduced-complexity approach to the detection and monitoring of static joint overloading in humans. In International Conference on Rehabilitation Robotics (ICORR).

Kimura et al., 1999. Kimura, H., Horiuchi, T., and Ikeuchi, K. (1999). Task-model based human robot cooperation using vision. In Intelligent Robots and Systems, 1999. IROS'99. Proceedings. 1999 IEEE/RSJ International Conference on, volume 2, pages 701-706. IEEE.

Klingspor et al., 1997. Klingspor, V., Demiris, J., and Kaiser, M. (1997). Human-robot communication and machine learning. Applied Artificial Intelligence, 11(7):719-746.

Kosuge et al., 1998. Kosuge, K., Hashimoto, S., and Yoshida, H. (1998). Human-robots collaboration system for flexible object handling. In Robotics and Automation, 1998. Proceedings. 1998 IEEE International Conference on, volume 2, pages 1841-1846. IEEE.

Kosuge and Kazamura, 1997a. Kosuge, K. and Kazamura, N. (1997a). Control of a robot handling an object in cooperation with a human. In Robot and Human Communication, 6th IEEE Intl. Workshop on, pages 142-147.

Kosuge and Kazamura, 1997b. Kosuge, K. and Kazamura, N. (1997b). Control of a robot handling an object in cooperation with a human. In Robot and Human Communication, 1997. RO-MAN'97. Proceedings., 6th IEEE International Workshop on, pages 142-147. IEEE.

Kronander and Billard, 2014. Kronander, K. and Billard, A. (2014). Learning compliant manipulation through kinesthetic and tactile human-robot interaction. IEEE transactions on haptics, 7(3):367380.

Krüger et al., 2009. Krüger, J., Lien, T. K., and Verl, A. (2009). Cooperation of human and machines in assembly lines. CIRP AnnalsManufacturing Technology, 58(2):628-646.

Kruse et al., 2015. Kruse, D., Radke, R. J., and Wen, J. T. (2015). Collaborative human-robot manipulation of highly deformable materials. In 2015 IEEE International Conference on Robotics and Automation (ICRA), pages 3782-3787. IEEE.

Kulic and Croft, 2007. Kulic, D. and Croft, E. A. (2007). Affective state estimation for human-robot interaction. IEEE Transactions on Robotics, 23(5):991-1000.

Lacevic and Rocco, 2011. Lacevic, B. and Rocco, P. (2011). Closedform solution to controller design for human-robot interaction. Journal of Dynamic Systems, Measurement, and Control, 133(2):024501-024501.

Lackey et al., 2011. Lackey, S., Barber, D., Reinerman, L., Badler, N. I., and Hudson, I. (2011). Defining next-generation multi-modal communication in human robot interaction. In Proceedings of the Human Factors and Ergonomics Society Annual Meeting, volume 55, pages 461-464. SAGE Publications.
Lallée et al., 2012. Lallée, S., Pattacini, U., Lemaignan, S., Lenz, A., Melhuish, C., Natale, L., Skachek, S., Hamann, K., Steinwender, J., Sisbot, E. A., et al. (2012). Towards a platform-independent cooperative human robot interaction system: Iii an architecture for learning and executing actions and shared plans. IEEE Transactions on Autonomous Mental Development, 4(3):239-253.

Lamy et al., 2009. Lamy, X., Colledani, F., Geffard, F., Measson, Y., and Morel, G. (2009). Achieving efficient and stable comanipulation through adaptation to changes in human arm impedance. In IEEE Int. Conf. on Robotics and Automation (ICRA'09), pages 265-271, Kobe, Japan.

Lawitzky et al., 2012a. Lawitzky, M., Medina, J., Lee, D., and Hirche, S. (2012a). Feedback motion planning and learning from demonstration in physical robotic assistance: differences and synergies. In Intelligent Robots and Systems (IROS), 2012 IEEE/RSJ Intl. Conf. on, pages 3646-3652.

Lawitzky et al., 2012b. Lawitzky, M., Medina, J. R., Lee, D., and Hirche, S. (2012b). Feedback motion planning and learning from demonstration in physical robotic assistance: differences and synergies. In 2012 IEEE/RSJ International Conference on Intelligent Robots and Systems, pages 3646-3652. IEEE.

Lecours et al., 2012. Lecours, A., Mayer-St-Onge, B., and Gosselin, C. (2012). Variable admittance control of a four-degree-of-freedom intelligent assist device. In Robotics and Automation (ICRA), 2012 IEEE International Conference on, pages 3903-3908. IEEE.

Lee and Ott, 2011. Lee, D. and Ott, C. (2011). Incremental kinesthetic teaching of motion primitives using the motion refinement tube. $A u$ tonomous Robots, 31(2-3):115-131.

Lee et al., 2007. Lee, S. Y., Lee, K. Y., Lee, S. H., Kim, J. W., and Han, C. S. (2007). Human-robot cooperation control for installing heavy construction materials. Autonomous Robots, 22(3):305-319.

Levratti et al., 2016. Levratti, A., De Vuono, A., Fantuzzi, C., and Secchi, C. (2016). Tirebot: A novel tire workshop assistant robot. In Advanced Intelligent Mechatronics (AIM), 2016 IEEE International Conference on, pages 733-738. IEEE.

Li et al., 2005. Li, Z., Hofemann, N., Fritsch, J., and Sagerer, G. (2005). Hierarchical modeling and recognition of manipulative gesture. In Proc. of the Workshop on Modeling People and Human Interaction at the IEEE Int. Conf. on Computer Vision, volume 77.

Maeda et al., 2001. Maeda, Y., Takahashi, A., Hara, T., and Arai, T. (2001). Human-robot cooperation with mechanical interaction based on rhythm entrainment-realization of cooperative rope turning. In Robotics and Automation, 2001. Proceedings 2001 ICRA. IEEE International Conference on, volume 4, pages 3477-3482. IEEE.

Magnanimo et al., 2014. Magnanimo, V., Saveriano, M., Rossi, S., and Lee, D. (2014). A bayesian approach for task recognition and future human activity prediction. In The 23rd IEEE International Symposium on Robot and Human Interactive Communication, pages 726-731. IEEE.

Magrini et al., 2014. Magrini, E., Flacco, F., and De Luca, A. (2014). Estimation of contact forces using a virtual force sensor. In 2014 IEEE/RSJ International Conference on Intelligent Robots and Systems, pages 2126-2133. IEEE.

Maurice et al., 2017. Maurice, P., Padois, V., Measson, Y., and Bidaud, P. (2017). Human-oriented design of collaborative robots. International Journal of Industrial Ergonomics, 57:88-102.

Medina et al., 2012a. Medina, J., Shelley, M., Lee, D., Takano, W., and Hirche, S. (2012a). Towards interactive physical robotic assistance: Parameterizing motion primitives through natural language. In RO-MAN, 2012 IEEE, pages 1097-1102.

Medina et al., 2012b. Medina, J. R., Shelley, M., Lee, D., Takano, W., and Hirche, S. (2012b). Towards interactive physical robotic assistance: Parameterizing motion primitives through natural language. In 2012 IEEE RO-MAN: The 21st IEEE International Symposium on Robot and Human Interactive Communication, pages 1097-1102. IEEE. 
Mittendorfer et al., 2015. Mittendorfer, P., Yoshida, E., and Cheng, G. (2015). Realizing whole-body tactile interactions with a selforganizing, multi-modal artificial skin on a humanoid robot. Advanced Robotics, 29(1):51-67.

Miyake and Shimizu, 1994. Miyake, Y. and Shimizu, H. (1994). Mutual entrainment based human-robot communication field-paradigm shift from human interface to communication field. In Robot and Human Communication, 1994. RO-MAN'94 Nagoya, Proceedings., 3rd IEEE International Workshop on, pages 118-123. IEEE.

Mojtahedi et al., 2017. Mojtahedi, K., Whitsell, B., Artemiadis, P., and Santello, M. (2017). Communication and inference of intended movement direction during humanhuman physical interaction. Frontiers in Neurorobotics, 11:21.

Morasso et al., 2007. Morasso, P., Casadio, M., Sanguineti, V., Squeri, V., and Vergaro, E. (2007). Robot therapy: the importance of haptic interaction. In 2007 Virtual Rehabilitation, pages 70-77.

Morel et al., 1998. Morel, G., Malis, E., and Boudet, S. (1998). Impedance based combination of visual and force control. In Robotics and Automation, 1998. Proceedings. 1998 IEEE International Conference on, volume 2, pages 1743-1748. IEEE.

Mörtl et al., 2012. Mörtl, A., Lawitzky, M., Kucukyilmaz, A., Sezgin, M., Basdogan, C., and Hirche, S. (2012). The role of roles: Physical cooperation between humans and robots. The International Journal of Robotics Research, 31(13):1656-1674.

Murphy, 2004. Murphy, R. R. (2004). Human-robot interaction in rescue robotics. IEEE Transactions on Systems, Man, and Cybernetics, Part C (Applications and Reviews), 34(2):138-153.

Nagai and Rohlfing, 2009. Nagai, Y. and Rohlfing, K. J. (2009). Computational analysis of motionese toward scaffolding robot action learning. IEEE Transactions on Autonomous Mental Development, 1(1):44-54.

Napier, 1956. Napier, J. R. (1956). The prehensile movements of the human hand. Bone \& Joint Journal, 38-B(4):902-913.

Newman, 1992. Newman, W. S. (1992). Stability and performance limits of interaction controllers. Journal of Dynamic Systems, Measurement, and Control, 114(4):563-570.

Nohama et al., 1995. Nohama, P., Lopes, A. V., and Cliquet, A. (1995). Electrotactile stimulator for artificial proprioception. Artificial organs, 19(3):225-230.

Ogata et al., 2003. Ogata, T., Masago, N., Sugano, S., and Tani, J. (2003). Interactive learning in human-robot collaboration. In Intelligent Robots and Systems, 2003.(IROS 2003). Proceedings. 2003 IEEE/RSJ International Conference on, volume 1, pages 162-167. IEEE.

Ott et al., 2006. Ott, C., Eiberger, O., Friedl, W., Bauml, B., Hillenbrand, U., Borst, C., Albu-Schaffer, A., Brunner, B., Hirschmuller, H., Kielhofer, S., et al. (2006). A humanoid two-arm system for dexterous manipulation. In Humanoid Robots, 2006 6th IEEE-RAS International Conference on, pages 276-283. IEEE.

Oztop et al., 2005. Oztop, E., Franklin, D. W., Chaminade, T., and Cheng, G. (2005). Human-humanoid interaction: is a humanoid robot perceived as a human? International Journal of Humanoid Robotics, 2(04):537-559.

Palunko et al., 2014. Palunko, I., Donner, P., Buss, M., and Hirche, S. (2014). Cooperative suspended object manipulation using reinforcement learning and energy-based control. In Intelligent Robots and Systems (IROS), 2014 IEEE/RSJ Intl. Conf. on, pages 885-891.

Pecchinenda, 1996. Pecchinenda, A. (1996). The affective significance of skin conductance activity during a difficult problem-solving task. Cognition \& Emotion, 10(5):481-504.

Perzanowski et al., 1998. Perzanowski, D., Schultz, A. C., and Adams, W. (1998). Integrating natural language and gesture in a robotics domain. In Intelligent Control (ISIC), 1998. Held jointly with IEEE International Symposium on Computational Intelligence in Robotics and Automation (CIRA), Intelligent Systems and Semiotics (ISAS), Proceedings, pages 247-252. IEEE.
Peternel and Babič, 2013. Peternel, L. and Babič, J. (2013). Learning of compliant human-robot interaction using full-body haptic interface. Advanced Robotics, 27(13):1003-1012.

Peternel et al., 2016a. Peternel, L., Noda, T., Petrič, T., Ude, A., Morimoto, J., and Babič, J. (2016a). Adaptive control of exoskeleton robots for periodic assistive behaviours based on EMG feedback minimisation. PLOS ONE, 11(2):e0148942.

Peternel et al., 2014. Peternel, L., Petrič, T., Oztop, E., and Babič, J. (2014). Teaching robots to cooperate with humans in dynamic manipulation tasks based on multi-modal human-in-the-loop approach. Autonomous robots, 36(1-2):123-136.

Peternel et al., 2016b. Peternel, L., Rozo, L., Caldwell, D., and Ajoudani, A. (2016b). Adaptation of robot physical behaviour to human fatigue in human-robot co-manipulation. In Humanoid Robots (Humanoids), 2016 IEEE-RAS International Conference on.

Peternel et al., 2016c. Peternel, L., Tsagarakis, N., and Ajoudani, A. (2016c). Towards multi-modal intention interfaces for human-robot co-manipulation. In Intelligent Robots and Systems (IROS), 2016 IEEE/RSJ International Conference on.

Petit et al., 2013. Petit, M., Lallée, S., Boucher, J.-D., Pointeau, G., Cheminade, P., Ognibene, D., Chinellato, E., Pattacini, U., Gori, I., Martinez-Hernandez, U., et al. (2013). The coordinating role of language in real-time multimodal learning of cooperative tasks. IEEE Transactions on Autonomous Mental Development, 5(1):3-17.

Piovesan et al., 2013. Piovesan, D., Morasso, P., Giannoni, P., and Casadio, M. (2013). Arm stiffness during assisted movement after stroke: The influence of visual feedback and training. IEEE Transactions on Neural Systems and Rehabilitation Engineering, 21(3):454465.

Plagemann et al., 2010. Plagemann, C., Ganapathi, V., Koller, D., and Thrun, S. (2010). Real-time identification and localization of body parts from depth images. In Robotics and Automation (ICRA), 2010 IEEE International Conference on, pages 3108-3113. IEEE.

Radford et al., 2015. Radford, N. A., Strawser, P., Hambuchen, K., Mehling, J. S., Verdeyen, W. K., Donnan, A. S., Holley, J., Sanchez, J., Nguyen, V., Bridgwater, L., et al. (2015). Valkyrie: Nasa's first bipedal humanoid robot. Journal of Field Robotics, 32(3):397-419. Ragaglia et al., 2016. Ragaglia, M., Zanchettin, A. M., Bascetta, L., and Rocco, P. (2016). Accurate sensorless lead-through programming for lightweight robots in structured environments. Robotics and Computer-Integrated Manufacturing, 39:9-21.

Rani et al., 2006. Rani, P., Liu, C., Sarkar, N., and Vanman, E. (2006). An empirical study of machine learning techniques for affect recognition in human-robot interaction. Pattern Analysis and Applications, 9(1):58-69.

Rani et al., 2004. Rani, P., Sarkar, N., Smith, C. A., and Kirby, L. D. (2004). Anxiety detecting robotic system-towards implicit humanrobot collaboration. Robotica, 22(01):85-95.

Reed, 2012. Reed, K. B. (2012). Cooperative physical human-human and human-robot interaction. In Peer, A. and Giachritsis, C., editors, Immersive Multimodal Interactive Presence. Springer Series on Touch and Haptic Systems.

Reed and Peshkin, 2008. Reed, K. B. and Peshkin, M. A. (2008). Physical collaboration of human-human and human-robot teams. IEEE Transactions on Haptics, 1(2):108-120.

Rosen et al., 2001. Rosen, J., Brand, M., Fuchs, M. B., and Arcan, M. (2001). A myosignal-based powered exoskeleton system. Systems, Man and Cybernetics, Part A: Systems and Humans, IEEE Transactions on, 31(3):210-222.

Rozo et al., 2015. Rozo, L., Bruno, D., Calinon, S., and Caldwell, D. G. (2015). Learning optimal controllers in human-robot cooperative transportation tasks with position and force constraints. In Intelligent Robots and Systems (IROS), 2015 IEEE/RSJ International Conference on, pages 1024-1030. IEEE.

Rozo et al., 2014. Rozo, L., Calinon, S., and Caldwell, D. G. (2014). Learning force and position constraints in human-robot cooperative transportation. In Robot and Human Interactive Communication, 
2014 RO-MAN: The 23rd IEEE International Symposium on, pages 619-624. IEEE.

Rozo et al., 2013. Rozo, L., Calinon, S., Caldwell, D. G., Jimenez, P., and Torras, C. (2013). Learning collaborative impedance-based robot behaviors. In AAAI Conference on Artificial Intelligence, pages 1422-1428, Bellevue, WA, USA.

Rozo et al., 2016. Rozo, L., Calinon, S., Caldwell, D. G., Jimenez, P., and Torras, C. (2016). Learning physical collaborative robot behaviors from human demonstrations. IEEE Transactions on Robotics, 32(3):513-527.

Sakita et al., 2004. Sakita, K., Ogawara, K., Murakami, S., Kawamura, K., and Ikeuchi, K. (2004). Flexible cooperation between human and robot by interpreting human intention from gaze information. In Intelligent Robots and Systems, 2004.(IROS 2004). Proceedings. 2004 IEEE/RSJ International Conference on, volume 1, pages 846-851. IEEE.

Sebanz et al., 2006. Sebanz, N., Bekkering, H., and Knoblich, G. (2006). Joint action: bodies and minds moving together. Trends in cognitive sciences, 10(2):70-76.

Shadmehr and Mussa-Ivaldi, 1994a. Shadmehr, R. and Mussa-Ivaldi, F. (1994a). Adaptive representation of dynamics during learning of a motor task. Journal of Neuroscience, 14(5):3208-3224.

Shadmehr and Mussa-Ivaldi, 1994b. Shadmehr, R. and Mussa-Ivaldi, F. A. (1994b). Adaptive representation of dynamics during learning of a motor task. The Journal of Neuroscience, 14(5):3208-3224.

Shannon, 1976. Shannon, G. (1976). A comparison of alternative means of providing sensory feedback on upper limb prostheses. Medical and biological engineering, 14(3):289-294.

Squeri et al., 2010. Squeri, V., Masia, L., Casadio, M., Morasso, P., and Vergaro, E. (2010). Force-field compensation in a manual tracking task. PLOS ONE, 5:1-12.

Strazzulla et al., 2017. Strazzulla, I., Nowak, M., Controzzi, M., Cipriani, C., and Castellini, C. (2017). Online bimanual manipulation using surface electromyography and incremental learning. IEEE Transactions on Neural Systems and Rehabilitation Engineering, 25(3):227-234.

Stulp et al., 2015. Stulp, F., Grizou, J., Busch, B., and Lopes, M. (2015). Facilitating intention prediction for humans by optimizing robot motions. In 2015 IEEE/RSJ International Conference on Intelligent Robots and Systems (IROS), pages 1249-1255.

Tamei and Shibata, 2011. Tamei, T. and Shibata, T. (2011). Fast reinforcement learning for three-dimensional kinetic human-robot cooperation with an emg-to-activation model. Advanced Robotics, 25(5):563-580.

Tan et al., 2009. Tan, J. T. C., Duan, F., Zhang, Y., Watanabe, K., Kato, R., and Arai, T. (2009). Human-robot collaboration in cellular manufacturing: design and development. In 2009 IEEE/RSJ International Conference on Intelligent Robots and Systems, pages 29-34. IEEE.

Tegin and Wikander, 2005. Tegin, J. and Wikander, J. (2005). Tactile sensing in intelligent robotic manipulation-a review. Industrial Robot: An International Journal, 32(1):64-70.

Todorov and Jordan, 2002. Todorov, E. and Jordan, M. I. (2002). Optimal feedback control as a theory of motor coordination. Nature neuroscience, 5(11):1226-1235.

Tomasello, 2009. Tomasello, M. (2009). Why We Cooperate. MIT Press.

Tsagarakis et al., 2016. Tsagarakis, N. G., Caldwell, D. G., Bicchi, A., Negrello, F., Garabini, M., Choi, W., Baccelliere, L., Loc, V., Noorden, J., Catalano, M., Ferrati, M., Muratore, L., Margan, A., Natale, L., Mingo, E., Dallali, H., Settimi, A., Rocchi, A., Varricchio, V., Pallottino, L., Pavan, C., Ajoudani, A., Lee, J., Kryczka, P., and Kanoulas, D. (2016). Walk-man: A high performance humanoid platform for realistic environments. Journal of Field Robotics (JFR).

Tsumugiwa et al., 2002a. Tsumugiwa, T., Yokogawa, R., and Hara, K. (2002a). Variable impedance control with virtual stiffness for human-robot cooperative peg-in-hole task. In Intelligent Robots and
Systems (IROS), 2002 IEEE/RSJ Intl. Conf. on, volume 2, pages $1075-1081$.

Tsumugiwa et al., 2002b. Tsumugiwa, T., Yokogawa, R., and Hara, K. (2002b). Variable impedance control with virtual stiffness for human-robot cooperative peg-in-hole task. In Intelligent Robots and Systems, 2002. IEEE/RSJ International Conference on, volume 2, pages 1075-1081. IEEE.

Ugur et al., 2015. Ugur, E., Nagai, Y., Celikkanat, H., and Oztop, E. (2015). Parental scaffolding as a bootstrapping mechanism for learning grasp affordances and imitation skills. Robotica, 33(05):11631180 .

Ulrich and Borenstein, 2001. Ulrich, I. and Borenstein, J. (2001). The guidecane-applying mobile robot technologies to assist the visually impaired. IEEE Transactions on Systems, Man, and CyberneticsPart A: Systems and Humans, 31(2):131-136.

Vanderborght et al., 2013a. Vanderborght, B., Albu-Schäffer, A., Bicchi, A., Burdet, E., Caldwell, D. G., Carloni, R., Catalano, M., Eiberger, O., Friedl, W., Ganesh, G., et al. (2013a). Variable impedance actuators: A review. Robotics and autonomous systems, 61(12):1601-1614.

Vanderborght et al., 2013b. Vanderborght, B., Albu-Schäffer, A., Bicchi, A., Burdet, E., Caldwell, D. G., Carloni, R., Catalano, M., Eiberger, O., Friedl, W., Ganesh, G., et al. (2013b). Variable impedance actuators: A review. Robotics and autonomous systems, 61(12):1601-1614.

Vogel et al., 2011. Vogel, J., Castellini, C., and Van Der Smagt, P. (2011). EMG-based teleoperation and manipulation with the DLR LWR-III. In Intelligent Robots and Systems (IROS), 2011 IEEE/RSJ Intl. Conf. on, pages 672-678.

Wakita et al., 2013. Wakita, K., Huang, J., Di, P., Sekiyama, K., and Fukuda, T. (2013). Human-walking-intention-based motion control of an omnidirectional-type cane robot. IEEE/ASME Transactions On Mechatronics, 18(1):285-296.

Wang et al., 1995. Wang, G., Zhang, X., Zhang, J., and Gruver, W. (1995). Gripping force sensory feedback for a myoelectrically controlled forearm prosthesis. In Systems, Man and Cybernetics, 1995. Intelligent Systems for the 21 st Century., IEEE International Conference on, volume 1, pages 501-504. IEEE.

Wang et al., 2005. Wang, Z., Liu, L., and Zhou, M. (2005). Protocols and applications of ad-hoc robot wireless communication networks: An overview. future, 10:20.

Wojtara et al., 2009. Wojtara, T., Uchihara, M., Murayama, H., Shimoda, S., Sakai, S., Fujimoto, H., and Kimura, H. (2009). Humanrobot collaboration in precise positioning of a three-dimensional object. Automatica, 45(2):333-342.

Yang et al., 2011. Yang, C., Ganesh, G., Haddadin, S., Parusel, S., Albu-Schaeffer, A., and Burdet, E. (2011). Human-like adaptation of force and impedance in stable and unstable interactions. IEEE Transactions on Robotics, 27(5):918-930.

Yang et al., 2016. Yang, C., Liang, P., Ajoudani, A., and Bicchi, A. (2016). Development of a robotic teaching interface for human to human skill transfer. In Intelligent Robots and Systems (IROS), IEEE International Conference on. IEEE.

Zanchettin et al., 2013a. Zanchettin, A. M., Bascetta, L., and Rocco, P. (2013a). Acceptability of robotic manipulators in shared working environments through human-like redundancy resolution. Applied ergonomics, 44(6):982-989.

Zanchettin et al., 2013b. Zanchettin, A. M., Bascetta, L., and Rocco, P. (2013b). Achieving humanlike motion: Resolving redundancy for anthropomorphic industrial manipulators. IEEE Robotics \& Automation Magazine, 20(4):131-138.

Zanchettin and Rocco, 2015. Zanchettin, A. M. and Rocco, P. (2015). Reactive motion planning and control for compliant and constraintbased task execution. In 2015 IEEE International Conference on Robotics and Automation (ICRA), pages 2748-2753. IEEE. 